\title{
THE LIZARDS AND AMPHISBAENIANS OF BOLIVIA (REPTILIA, SQUAMATA): CHECKLIST, LOCALITIES, AND BIBLIOGRAPHY
}

\author{
L. Dirksen $(*)$ and I. De la Riva $(* *)$
}

\begin{abstract}
The number of Bolivian lizards and amphisbaenids is increased from 79 (Fugler, 1989) to 102 in this updated and comprehensive list, which includes all new species and taxonomic changes published recently, as well as locality data. Coordinates for the localities are provided in a locality list. Three species are reported herein for the first time: Kentropyx pelviceps, Liolaemus schmidti, and Uranoscodon superciliosus.
\end{abstract}

Key words: Saurians, Amphisbaenians, distribution, bibliography, Bolivia.

\section{RESUMEN}

Los saurios y anfisbénidos de Bolivia (Reptilia, Squamata): lista patrón, localidades y bibliografía

El número de saurios y anfisbénidos conocidos en Bolivia se incrementa de 79 especies (Fugler, 1989) a 102 en la presente lista actualizada, que incorpora todas las especies nuevas, los cambios taxonómicos recientes y todas las localidades de colecta conocidas con sus coordenadas. Tres especies son citadas para Bolivia por primera vez en este trabajo: Kentropyx pelviceps, Liolaemus schmidti y Uranoscodon superciliosus.

Palabras clave: Saurios, Anfisbénidos, distribución, bibliografía, Bolivia.

\section{Introduction}

Bolivia lies in the middle of South America, between the high Andes in the West, the hot and wet Amazonia in the North and the dry Chaco in the South. These conditions account for a large variety of different microclimates and ecosystems with their particular flora and fauna. The biological diversity of Bolivia is still poorly known, even at a lesser extent than that of other South American countries. This fact affects not only to invertebrates but to vertebrates as well. Among these, there is a remarkable lack of information on reptiles, especially Squamata. Fugler (1989) presented a preliminar checklist of lizards and amphisbaenians, in which he reported 79 species. As a result of our own investigations in Bolivia, research in museum collections (through direct examination of specimens and critical evaluation of collection catalogues) and an extensive literature work, we can now increase substantially the number of species of Bolivian lizards and amphisbaenians. In this paper we present an updated list of the lizards and amphisbaenians of Bolivia, the localities where they are known, and a comprehensive bibliography on this topic. Probably, several references containing additional localities might have been overlooked, but, overall, we hope this list is a useful tool for anybody interested in Bolivian herpetology.

* Zoologisches Forschungsinstitut und Museum Alexander Koenig, Adenauerallee 160, 53113 Bonn, Germany.

** Museo Nacional de Ciencias Naturales, CSIC, C/ José Gutiérrez Abascal 2, 28006 Madrid, Spain. 


\section{Material and methods}

All data are based on published records or voucher specimens. No records from field observations are given unless specimens were collected. If we did not examine the specimens but based our work on a museum collection catalogue, we only accepted localities as valid if they are near to other localities known for the species considered, or at least they fall in the same general type of ecosystem. We did not include new species for Bolivia based solely on collection catalogues if we have any doubt about their determination or locality. The locality data from museum unpublished catalogues were some times hand-written and often the data are vague, misspelled and usually coordinates are missing. Obvious misspellings were corrected. Localities too imprecise (i. e., "Alto Beni", "Los Yungas", "Province Sara", "Cordillera Tres Cruces", "Altiplano", etc.) usually are not listed in the gazeteer (Appendix 1); those that could not be found are indicated by an asterisk (*). Usually, coordinates given as kilometric distances from a given point (i. e., a river mouth or town) are not provided. Instead, we provide coordinates of the locality of reference. Fig. 1 shows the situation of Bolivia in the continent and its political divisions.

We used the name Río Iténez instead Río Guaporé for the river on the Bolivian and Brazil boundary. Both names are equally found in literature and maps.

The species are sorted whitin the genus by alphabetical order and the genus in the same way within families. We follow the arrangement proposed by Frost \& Etheridge (1989) for the old family Iguanidae. In agreement with Avila-Pires (1995), we do not follow the arrangement proposed by Guyer \& Savage (1986, Syst. Zool., 35: 509-531) for anoles.

Geographic records are listed by Bolivian departments (underlined), which are arranged in alphabetic order. A citation consists of a locality and its source (an author or a museum acronym), separated by a comma, and it is finished by a point. The citations are arranged by chronological order when they come from published data and by alphabetical order of museum acronyms when they come from collections. A semicolon is between localities, if more than one locality is cited from the same source. We used a comma if the locality is briefly described (i. e., San Antonio, Río Mapiri). Citations have been translated if they were originally in a language other than English and included more or less long sentences.

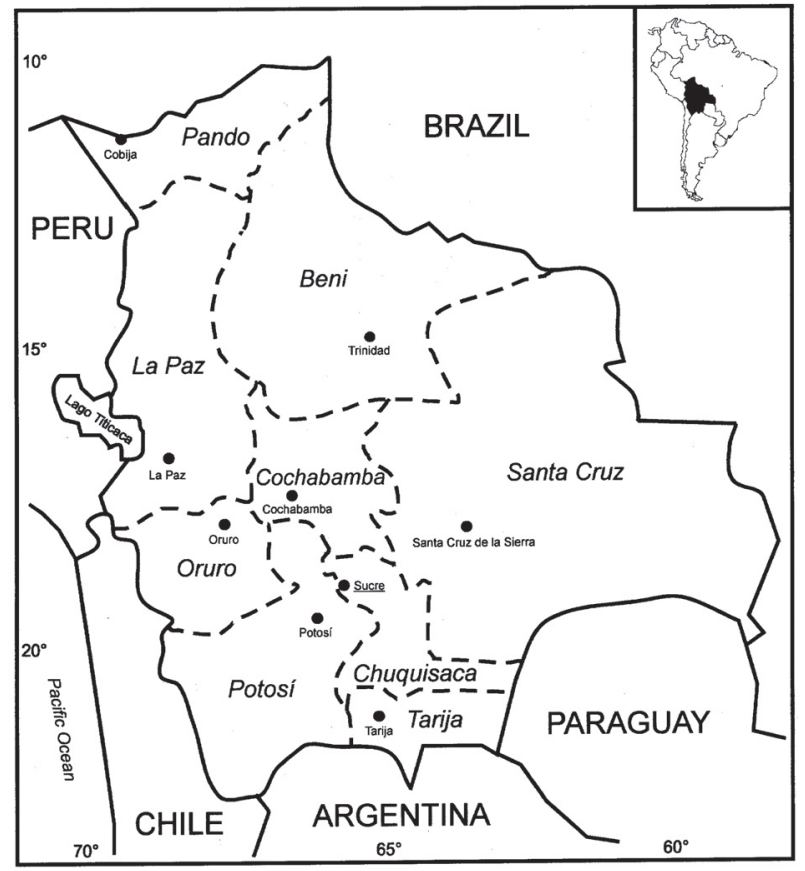

Fig. 1.- Map of Bolivia showing the nine departments and their capitals.

Fig. 1.- Mapa de Bolivia donde se indican los nueve departamentos y sus capitales.

Published data for a given department are reported first, followed by museum data. When for a species more than one record was found for the same locality (for example, both in the literature and museum collections), we used it only once. In this case, we always preferred to give credit to an author rather than using museum information. Likewise, in the case that a record was provided both in an unpublished thesis or report and in a publication, we have given priority to the publication irrespective of which document was the oldest. When more than one author provided the same record, we gave preference to the oldest publication. Only the first acronym museum in alphabetical order is used in the case of having the same data from more than one museum. We have tracked the history of taxonomic changes of the species and subespecies considered herein, but we do not offer lists of synonyms to avoid making this paper extremely long. Thus, it can appear a citation in which the species and the authorship are different from those of the original citation. For example, Peracca (1897) cited Amphisbaena camura Cope at Caiza; 
this population is presently considered as belonging to the species A. bolivica Mertens. Thus, the record of $A$. bolivica at Caiza is herein credited to Peracca, despite of the fact that in Peracca's publication, the name $A$. bolivica does not appear. Likewise, if the name of a locality changed through time, we have used the current name.

Bibliographic references listed at the end of this paper intend to be exhaustive and not limited to those cited in the main text. References containing the original descriptions of all the species considered are included, as well as references dealing with Bolivian saurians but providing no locality data.

Museum acronyms follow Leviton et al., (1985, Copeia 1985: 802-832). Other institutions not reported therein are:

CBS- Colección Boliviana de Fauna (before: IE- Instituto de Ecología plus Museo Nacional de Historia Natural), La Paz, Bolivia

CET- Centro de Estudios Tropicales, Sevilla, Spain

MNK- Museo de Historia Natural "Noel Kempff Mercado", Santa Cruz de la Sierra, Bolivia

Abbreviations used: Dpto. $=$ departamento (department); $\mathrm{N}=$ North; $\mathrm{E}=$ East; $\mathrm{S}=$ South; $\mathrm{W}=$ West; $\mathrm{ca} .=$ circa; $\mathrm{Cd}=$ Ciudad (city); $\mathrm{ft}=$ feet; $\mathrm{km}=$ kilometers; $\mathrm{m}=$ meters; $\mathrm{mi}=$ miles.

\section{The amphisbaenians and lizards of Bolivia}

\section{AMPHISBAENIDAE}

\section{Amphisbaena alba Linnaeus, 1758}

Beni: Río Mamoré (Cope, 1862a). Guayaramerín (Fugler, 1984). La Paz: Upper Río Beni (Cope, 1885). Santa Cruz: Province Sara (Griffin, 1917). Esperanza (Procter, 1921). $2 \mathrm{~km}$ from Concepción on San Ignacio road, CET. Santiago, FMNH. Santa Cruz de la Sierra, MNK. Buena Vista; Río Surutú, UMMZ. Airport Viru Viru, ZFMK. Tarija: Villa Montes, ZFMK.

\section{Amphisbaena angustifrons Cope, 1861}

Beni: San Joaquín, FMNH. Cochabamba: Río Ichilo, ca. $54 \mathrm{~km} \mathrm{~S}$ Boca Chapare, AMNH. Tarija: Upper Río Pilcomayo (Parker, 1928a). Ca. Villa Montes (Hellmich, 1960).

Amphisbaena bolivica Mertens, 1929

Beni: Riberalta (Montero, 1996). Estancia El Porvenir, ZFMK. Santa Cruz: Buenavista (Gans, 1965). Tarija: Caiza (Peracca, 1897). Villa Montes (Mertens, 1929).

\section{Amphisbaena camura Cope, 1862}

Beni: "Headwaters of the Amazon river, Bolivia" (Werner, 1910). San Joaquín, FMNH. Magdalena, USNM. El Porvenir,
ZFMK. Santa Cruz: San José de Chiquitos (Gans, 1960). Buena Vista (Hellmich, 1960). Santa Cruz de la Sierra (Gans, 1965). Parque Nacional Amboró (Lavilla et al., 1996). Pampa Grande (Montero, 1996). Tarija: Caiza; Villa Montes (Hellmich, 1960).

Amphisbaena cegei Montero, Sáfadez \& Álvarez, 1997

Santa Cruz: Pampa Grande (Montero et al., 1997).

Amphisbaena darwini Duméril \& Bibron, 1839

La Paz: Sorata (Boettger, 1891). San Antonio, Río Mapiri (Werner, 1910). Tarija: Caiza (Peracca, 1897).

\section{Amphisbaena fuliginosa Linnaeus, 1758}

Chuquisaca: Charobamba (Rhodes, 1963). Cochabamba: Villa Tunari, MNK. La Paz: Sorata (Boettger, 1891). LoS Yungas; Tipuani (Rhodes, 1963). Santa Cruz: Puerto Almacén (De la Riva, 1993). Campamento, Río San Martín (Montero et al., 1995). Los Fierros (Scrocchi \& Gonzáles, 1996).

Amphisbaena silvestrii Boulenger, 1902 Santa Cruz: Buena Vista (Gans, 1964).

\section{Amphisbaena slateri Boulenger, 1907}

La Paz: Pelecnes (= Pelechuco), NE Titikaka Lake; San Antonio, Río Mapiri (Gans, 1967).

\section{Amphisbaena vermicularis Wagler, 1824}

Santa Cruz: San Ignacio de Velasco (Hellmich, 1960). Tarija: Villa Montes, ZFMK.

\section{Cercolophia borelli (Peracca, 1897)}

Santa Cruz: Finca de Milanos; Santa Cruz de la Sierra (Montero et al., 1995). Cupesí (Gonzáles, 1998). Tarija: Caiza (Peracca, 1897).

\section{Cercolophia steindachneri (Strauch, 1883)}

Santa Cruz: Puerto Almacén (De la Riva, 1993). El Refugio (Harvey, 1995).

\section{Leposternon microcephalum Wagler, 1824}

Cochabamba: Villa Tunari, KU. Santa Cruz: Province Sara (Griffin, 1917). Buena Vista (Fugler, 1989). Parque Nacional Amboró; Yapacaní (Lavilla et al., 1996). Río Surutú, UMMZ.

\section{ANGUIDAE}

Diploglossus fasciatus (Gray, 1831)

Beni: Cachuela Esperanza, AMNH. La Paz: Sorata (Boettger, 1891). Confluence of the Río Kaka and the Río Beni (Barbour, 1909). Alto Beni, ANSP.

\section{Ophiodes intermedius Boulenger, 1894}

Beni: Rancho "San Marcos", in the confluence of the Isiboro and Ichoa rivers (Fugler, 1989). Cochabamba: 
Cochabamba (Werner, 1910). Santa Cruz: El Fuerte (Dirksen et al., 1995). Pampas Unión (Montero et al., 1995). Vallegrande, MNK.

Ophiodes yacupoi Gallardo, 1966

Santa Cruz: Guadalupe (Montero et al., 1995).

\section{GEKKONIDAE}

\section{Gonatodes hasemani Griffin, 1917}

Beni: Villa Bella (Griffin, 1917). Tumi Chucua (Fugler, 1983). Ivon, AMNH. Pando: Ingavi, on N bank of Río Orthon; San Juan del Nuevo Mundo, USNM.

\section{Gonatodes humeralis Guichenot, 1855}

Beni: Villa Bella (Griffin, 1917). Tumi Chucua (Fugler, 1983). Ivon, AMNH. Pando: Abuná (Schmidt \& Inger, 1951). Ingavi, on N bank of Río Orthon, USNM. Santa Cruz: Puerto Almacén (Montero et al., 1995). Parque Nacional Noel Kempff Mercado (Harvey et al., 1998). San José de Chiquitos, ZFMK.

Hemidactylus mabouia (Moreau de Jonnès, 1818) Beni: Río Mamoré (Kluge, 1969). Guayaramerín (Fugler, 1984). Pando: Cobija (Fugler, 1984). $25 \mathrm{~km} \mathrm{~S}$ of Cobija (Fugler, 1988a). Santa Cruz: Concepción (Dirksen, 1995). Pampa Grande; Samaipata (Lavilla et al., 1996). Flor de Oro (Scrocchi \& Gonzáles, 1996). Santa Cruz de la Sierra, CET.

Homonota dorbignyi (Duméril \& Bibron, 1836)

Chuquisaca: "Along Río Grande at Pampa Ruiz (between Valle Grande and Pescado)" (Duméril \& BIBRON, 1836).

Homonota fasciata (Duméril \& Bibron, 1836)

Cochabamba: Dpto. Cochabamba, UMMZ. Santa Cruz: Huasacañada (Montero et al., 1995). Pampa Grande (Lavilla et al., 1996). Cerro Colorado; La Madre; Yapiroa (Gonzáles, 1998). San José de Chiquitos, AMNH. Tarija: Aguairenda; Caiza (Peracca, 1897).Villa Montes (Mertens, 1929). Andes of Catamarca (Hellmich, 1960).

Lygodactylus wetzeli Smith, Martin \& Swain, 1977

Santa Cruz: Cerro Colorado; Cupesí; Tierras Nuevas (Gonzáles, 1998). Charagua, AMNH. Tarija: ca. Villa Montes (Fugler, 1989).

Phyllopezus goyazensis Peters, 1887

Santa Cruz: Puerto Suárez (Griffin, 1917). Tarija: San Francisco (=Villa Montes) (Peracca, 1897).

\section{Phyllopezus pollicaris (Spix, 1825)}

Santa Cruz: Puerto Suárez (Griffin, 1917). El Carmen; El Portón; 3 km S of Roboré; San José de Chiquitos (Gans, 1960). Buena Vista; San Ramón, N of San José de Chiquitos (Hellmich, 1960). Inselberg (Dirksen, 1995). Parque Nacional Amboró (Lavilla et al., 1996). Cerro Colorado; Cupesí; La
Madre; Tierras Nuevas; Yapiroa (Gonzáles, 1998). San José de Chiquitos, AMNH. Santa Cruz, CAS. Santiago, FMNH. Tarija: Villa Montes (Hellmich, 1960).

\section{Thecadactylus rapicauda (Houttuyn, 1782)}

Beni: Tumi Chucua (Fugler, 1983). Río Quiquibey (Fugler, 1984). Río Yata (on road from Guayaramerín to Cachuela Esperanza), AMNH. La Paz: Tumupassa, AMNH. Pando: Abuná (Schmidt \& Inger, 1951). $25 \mathrm{~km} \mathrm{~S}$ of Cobija (Fugler, 1984). Río Madre de Dios, ZMH.

\section{GYMNOPHTHALMIDAE}

Bachia dorbignyi (Duméril \& Bibron, 1839)

Beni: Reyes (Boulenger, 1898). Estancia El Porvenir (De la Riva et al., 1992). Río Curiraba, Beni Biosphere Reserve, USNM. Cochabamba: Villa Tunari, KU. La Paz: Barraca, Río Madidi; Misiones Mosetenes (Boulenger, 1898). Tumupassa (Burt \& Burt, 1931). Pando: Reserva Nacional Amazónica Manuripi-Heath (Aparicio, 1992). Santa Cruz: Santa Cruz de la Sierra (Duméril \& Bibron, 1839). Las Juntas (Griffin, 1917). Esperanza (Procter, 1921). Buena Vista (Parker, 1928b). Río Surutú (Dixon, 1973). San Pablo (Dirksen, 1995). Parque Nacional Amboró (Lavilla et al., 1996). Yapiroa (Gonzáles, 1998). Lomas de Arena, CET.

\section{Cercosaura ocellata Wagler, 1830}

La Paz: Tumupassa (Ruibal, 1952). Pando: Reserva Nacional Amazónica Manuripi-Heath (Aparicio, 1992). Santa Cruz: Province Sara (Griffin, 1917). Río Surutú (Ruibal, 1952). Buena Vista; Parque Nacional Amboró; San Juan del Potrero (Lavilla et al., 1996).

\section{Iphisa elegans Gray, 1851}

Cochabamba: Dpto. Cochabamba (Dixon, 1974). Puerto San Mateo (Avila-Pires, 1995). Santa Cruz: Parque Nacional Noel Kempff Mercado (Harvey et al., 1998).

Micrablepharus maximiliani Reinhardt \& Lütken, 1862

Santa Cruz: Cerro Colorado (Gonzáles, 1998).

\section{Neusticurus ecpleopus Cope, 1876}

Bolivia: Río Beni (Werner, 1910). La Paz: Misiones Mosetenes (Boulenger, 1898). "Yungas, ca. 1000-1800 m (sic), Río Suapi and Songo" (Werner, 1910). $1.6 \mathrm{~km} \mathrm{~W}$ of Puerto Linares (Fugler, 1984). Ca. $40 \mathrm{~km}$ from Caranavi to Beni, Serranía Bellavista, CET. Santa Cruz: Río Saguayo, Parque Nacional Amboró (Montero et al., 1995). Buena Vista; Parque Nacional Amboró (Lavilla et al., 1996).

Neusticurus ocellatus Sinitsin, 1930

Beni: Rurrenabaque (Sinitsin, 1930).

\section{Opipeuter xestus Uzzell, 1969}

Chuquisaca: El Palmar (Harvey, 1997). Cochabamba: Incachaca; Locotal; Monte Punco; Paracti; Yungas de 
Cochabamba; Yungas del Palmar (Uzzell, 1969). Sehuencas (Köhler et al., 1995). La Siberia; Río Chua Khocha, CET; Tablasmontes, MNK. Tucachaca, UMMZ. Santa Cruz: Remates, N of San Juan del Potrero, ZFMK.

\section{Pantodactylus schreibersii (Wiegmann, 1834)}

Beni: Rurrenabaque (Ruibal, 1952). Estación Biológica Beni; Rancho "San Marcos", in the confluence of the Río Isiboro and Río Ichoa (Fugler, 1989). Guayaramerín; Santa Rosa, AMNH. San Joaquín, FMNH. Chuquisaca: El Palmar (Harvey, 1997). Cochabamba: Boca Chapare; 4 km S Boca Chapare; 54 km S Boca Chapare, AMNH. La Paz: Chulumani; Coroico (Boulenger, 1898). Ixiamas; Tumupassa (Ruibal, 1952). $15 \mathrm{~km}$ before Chulumani; ca. $1 \mathrm{mi} \mathrm{W}$ of Puerto Linares; Yanacachi, USNM. Santa Cruz: Buena Vista (Burt \& Burt, 1931). El Fuerte (Dirksen et al., 1995). El Refugio (Harvey, 1995). Pampa Grande; Parque Nacional Amboró (Lavilla et al., 1996). Samaipata, CET. Santa Cruz de la Sierra, MNK. Río Surutú, UMMZ. Tarija: Caiza; San Francisco (Peracca, 1897). San Francisco, Upper Pilcomayo River (Hellmich, 1960). Sunchal (Fugler, 1989).

\section{Prionodactylus argulus (Peters, 1862)}

Cochabamba: Monterredondo (Uzzell, 1973). La Paz: San Fermín (Andersson, 1914). Santa Cruz: Buena Vista (Uzzell, 1973). Parque Nacional Amboró (Lavilla et al., 1996).

\section{Prionodactylus eigenmanni Griffin, 1917}

Beni: Rancho Espíritu, Río Yacuma; Rurrenabaque (Uzzell, 1973). Pando: San Juan del Nuevo Mundo, USNM. Santa Cruz: Province Sara (Griffin, 1917). Perseverancia (Hoogmoed \& Vaca, 1990). Buena Vista (Avila-Pires, 1995). Parque Nacional Amboró (Lavilla et al., 1996).

Prionodactylus manicatus (O'Shaughnessy, 1881)

Beni: Rancho San Marcos, in the confluence of the Isiboro and Ichoa rivers, (Fugler, 1989). Cochabamba: Yungas de Cochabamba $(2200 \mathrm{~m})$, UMMZ. La Paz: Chaco (Werner, 1899).

Proctoporus bolivianus Werner, 1910 1914)

La Paz: Sorata (Werner, 1910). Pelechuco (Andersson,

Proctoporus guentheri (Boettger, 1891)

Cochabamba: Cochabamba, $2516 \mathrm{~m}$; Yungas del Palmar (Uzzell, 1970). La Paz: Sorata (Boettger, 1891). Calabatea (Emmons, 1991). Santa Cruz: El Fuerte (Dirksen et al., 1995).

\section{Ptychoglossus brevifrontalis Boulenger, 1912}

Cochabamba: Cochabamba Valley (Harris, 1994).

Vanzosaura rubricauda (Boulenger, 1902)

Beni: Dpto. Beni (Werner, 1910). Cochabamba: Dpto. Cochabamba, UMMZ. Santa Cruz: Cerro Colorado (Gonzáles, 1998). San Antonio de Parapetí, AMNH. Tarija: Villa Montes (Hellmich, 1960).

\section{HOPLOCERCIDAE}

Enyalioides palpebralis (Boulenger, 1883)

Bolivia: NW Bolivia (Avila-Pires, 1995).

\section{Hoplocercus spinosus Fitzinger, 1843}

Santa Cruz: El Refugio (Harvey, 1995). $10 \mathrm{~km} \mathrm{~S}$ of Colonia San Martín (Scrocchi \& Gonzáles, 1996).

\section{IGUANIDAE}

\section{Iguana iguana (Linnaeus, 1758)}

Beni: Tumi Chucua (Fugler, 1985). Arroyo Grande; $8 \mathrm{~km}$ $\mathrm{N}$ of Exaltación; Guayaramerín; $20 \mathrm{~km} \mathrm{~W}$ of La Rangeira; Puerto Cap. Vázquez; Puerto Moré; Río Mamoré (1259' S); Santa Fé, Río Iténez, AMNH. Riberalta, UMMZ. Pando: Abuná (Schmidt \& Inger, 1951). Reserva Nacional Amazónica Manuripi-Heath (Aparicio, 1992). Santa Cruz: Florida (De la Riva et al., 1992). Flor de Oro (Scrocchi \& Gonzáles, 1996).

\section{POLYCHROTIDAE}

\section{Anolis fuscoauratus D'Orbigny, 1837}

Beni: Río Mamoré, between Loreto and the mouth of Río Sara (D'Orbigny, 1847). "Province of Moxos" (Bocourt, 1873). Estación Biológica Beni (Fugler, 1989). Río Mamoré, $10 \mathrm{~km}$ E of San Antonio; Santa Rosa, AMNH. Cochabamba: $40 \mathrm{~km}$ from Villa Tunari to Cochabamba, CET. Valle de Sajta, MNK. Locotal; Yungas de Cochabamba, UMMZ. La Paz: Sorata (Boettger, 1891). Ca. $1.6 \mathrm{~km} \mathrm{~W}$ of Puerto Linares (Fugler, 1984). Mapiri, AMNH. Santa Cruz: Buena Vista (Barbour, 1934). Parque Nacional Amboró (Lavilla et al., 1996). $2 \mathrm{~km} \mathrm{~N}$ of Boca Chapare, AMNH. Río Surutú, UMMZ.

\section{Anolis meridionalis Boettger, 1885}

Beni: Estancia Yutiole, $20 \mathrm{~km} \mathrm{~S}$ of San Joaquín; $23 \mathrm{~km} \mathrm{~W}$ of San Javier; $10 \mathrm{~km} \mathrm{~W}$ of San Pedro, AMNH. Santa Cruz: Province Sara (Griffin, 1917). Buena Vista (Barbour, 1934). Parque Nacional Amboró (Lavilla et al., 1996). Río Surutú, UMMZ.

\section{Anolis ortonii Cope, 1868}

Beni: Tumi Chucua (Fugler, 1983). La Paz: Chulumani; Coroico (Boulenger, 1898).

\section{Anolis punctatus Daudin, 1802}

Beni: Rurrenabaque; Santa Rosa, Río Mamoré (Fugler, 1988b). La Paz: Río Chimate (Fugler, 1988b). Pando: ca. 12 $\mathrm{km}$ by road $\mathrm{S}$ of Cobija and $8 \mathrm{~km}$ from Mucden (Fugler, 1988b). Santa Cruz: Dpto. Santa Cruz de la Sierra (Griffin, 1917). Buena Vista (Fugler, 1988b). Puerto Almacén (De la Riva, 1993). Parque Nacional Amboró (Lavilla et al., 1996).

\section{Anolis scapularis Boulenger, 1908}

Santa Cruz: Province Sara (Boulenger, 1908). Parque Nacional Amboró (Lavilla et al., 1996). 


\section{Polychrus acutirostris Spix, 1825}

Beni: Rancho San Marcos, in the confluence of the Isiboro and Ichoa rivers (Fugler, 1989). Estancia Edén, $25 \mathrm{~km} \mathrm{SW}$ of San Joaquín; Estancia Yutiole, $20 \mathrm{~km} \mathrm{~S}$ of San Joaquín; Trinidad, AMNH. Chuquisaca: El Salvador (Montero et al., 1995). Cochabamba: Dpto. Cochabamba, UMMZ. Santa Cruz: Asunción; Province Sara; Puerto Suárez (Griffin, 1917). Buenavista (Hellmich, 1960). Parque Nacional Amboró; Yapacaní (Lavilla et al., 1996). San José de Chiquitos, AMNH. Santiago, FMNH. Río Surutú, UMMZ. Tarija: Aguairenda; Caiza (Peracca, 1897). Villa Montes (Hellmich, 1960).

\section{Polychrus liogaster Boulenger, 1908}

Beni: Tumi Chucua (Fugler, 1983). Rurrenabaque (Fugler, 1984). Río Curichá (1 km from the confluence with Río Iténez) Santa Rosa, AMNH. Cochabamba: Dpto. Cochabamba, AMNH. Santa Cruz: Province Sara (Boulenger, 1908). Buena Vista (Avila-Pires, 1995). Ca. $40 \mathrm{~km}$ from San Javier to Concepción, CET. Parque Nacional Amboró; Warnes, MNK. Río Surutú, UMMZ.

Urostrophus gallardoi Etheridge \& Williams, 1991

Santa Cruz: Santa Cruz de la Sierra (Etheridge \& Williams, 1991). Tarija: Border of Tarija river, entry to Salinas coming from Potrerillos, Reserva Nacional de Flora y Fauna de Tariquía (Torres, 1996).

\section{SCINCIDAE}

\section{Mabuya bistriata (Spix, 1825)}

Beni: Reyes (Boulenger, 1898). Tumi Chucua (Fugler, 1983). Rancho San Marcos, in the confluence of the Isiboro and Ichoa rivers (Fugler, 1989). $10 \mathrm{~km} \mathrm{~W}$ of Trinidad, ZFMK. La Paz: Misiones Mosetenes (Boulenger, 1898). $1.6 \mathrm{~km} \mathrm{~W}$ of Puerto Linares (Fugler, 1984). Pando: Abuná (Schmidt \& Inger, 1951). Santa Cruz: El Carmen (Gans, 1960). El Refugio (Harvey, 1995).

\section{Mabuya cochabambae Dunn, 1935}

Cochabamba: Pocona (Dunn, 1935). Ca. $100 \mathrm{~km}$ from Cochabamba to Santa Cruz, 3800 m (De la Riva et al., 1992). Santa Cruz: Dpto. Santa Cruz (Dunn, 1935).

\section{Mabuya dorsovittata Cope, 1862 \\ Santa Cruz: Puerto Suaréz (Hellmich, 1960).}

\section{Mabuya frenata (Cope, 1862)}

Beni: Reyes; Riberalta; Río Beni (Dunn, 1935). Rancho San Marcos, in the confluence of the Isiboro and Ichoa rivers (Fugler, 1989). Puerto Almacén, AMNH. San Joaquín, FMNH. Riberalta, UMMZ. Cochabamba: Tiraque (Dunn, 1935). La Paz: Ixiamas (Dunn, 1935). Santa Cruz: Buena Vista (Dunn 1935). El Carmen (Gans, 1960). San José de Chiquitos (Hellmich, 1960). Puerto Almacén (De la Riva, 1993). Santa Rosa de la Roca (Dirksen, 1995). Abra de la Cruz (Lavilla et al., 1996). Flor de Oro; Meseta de Caparuch (Scrocchi \& Gonzáles, 1996). La Madre; Tierras Nuevas; Yapiroa
(Gonzáles, 1998). Santa Elena; Santiago, FMNH. Tarija: Aguairenda; Caiza; San Francisco (Peracca, 1897). Taringuiti, UMMZ.

\section{Mabuya guaporicola Dunn, 1935}

Beni: Rancho San Marcos, in the confluence of the Isiboro and Ichoa rivers (Fugler, 1989). Santa Cruz: Dpto. Santa Cruz (Peters \& Donoso-Barros, 1970). Parque Nacional Noel Kempff Mercado (Harvey et al., 1998). Buena Vista, UMMZ.

Mabuya nigropalmata Andersson, 1918

La Paz: San Fermín (Andersson, 1918).

\section{Mabuya nigropunctata (Spix, 1825)}

Santa Cruz: Inselberg (Dirksen, 1995). Parque Nacional Noel Kempff Mercado (Harvey et al., 1998).

\section{TEIIDAE}

Ameiva ameiva (Linnaeus, 1758)

Beni: Reyes (Boulenger, 1898). Trinidad (Burt \& Burt, 1930). Tumi Chucua (Fugler, 1983). Rurrenabaque (Fugler, 1984). Estancia Venecia; San Borja (De la Riva et al., 1992). Camiaco; Chacabo Indian Village; Costa Márquez; El Remanso; Guayaramerín; Ivón; Pacusal; Pampa de Meio; Puerto Almacén; Puerto Caballo; Puerto Cap. Vázquez; Puerto

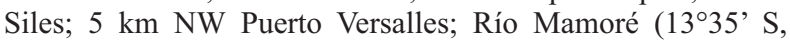
$\left.12^{\circ} 26^{\prime} \mathrm{S}\right) ; 10 \mathrm{~km}$ E San Antonio; $10 \mathrm{~km}$ W San Pedro; Santa Fé; Santa Rosa, AMNH. San Antonio; San Joaquín, FMNH. Estancia La Cabaña (1 km E of Emboscada); Riberalta, UMMZ. Beni Biosphere Reserve; Río Iténez, Lago Victoria, USNM. Cochabamba: Villa Tunari (De la Riva et al., 1992). Boca Chapare, AMNH. Puerto San Francisco, $35 \mathrm{~km} \mathrm{~N}$ Chipiriri, KU. La Paz: Misiones Mosetenes (Boulenger, 1898). $1.6 \mathrm{~km} \mathrm{~W}$ of Puerto Linares (Fugler, 1984). Río Chimate (Fugler, 1989). Upper Río Madidi; Río Machariapo (Emmons, 1991). Pampas del Heath (Pérez, 1997). Huachi; Río Mapiri, AMNH. 4 km NW Alcoche; Saroria, UMMZ. Pando: Abuná (Schmidt \& Inger, 1951). Reserva Nacional Amazónica Manuripi-Heath (Aparicio, 1992). Río Nareuda, AMNH. Cobija, ZFMK. Santa Cruz: Province Sara (Griffin, 1917). Esperanza (Procter, 1921). Buena Vista (Parker, 1928b). Perseverancia (Hoogmoed \& Vaca, 1990). Comunidad Guirapembi; 38 km E of Florida; Los Fierros (De la Riva et al., 1992). Puerto Almacén (De la Riva, 1993). Florida; Inselberg (Dirksen, 1995). El Refugio (Harvey, 1995). Ayacucho de Yapacaní; Campamento Macuñucú; La Guitarra; Parque Nacional Amboró (Lavilla et al., 1996). Aguaraigua; Cerro Colorado; Cupesí; La Madre; Tierras Nuevas; Yapiroa (Gonzáles, 1998). Sounaiporta (= Samaipata?), AMNH. Cotoca; Okinawa 1, KU. Piso Firme; San Javier; Santa Cruz de la Sierra, MNK. Buena Vista; Río Surutú; neighborhood of Santa Cruz de la Sierra, UMMZ. Ayacucho, 16 km SW Santa Cruz, USNM. Tarija: Aguairenda; San Francisco (Peracca, 1897). Fortín Magariños (Mertens, 1929).

\section{Ameiva vittata (Boulenger, 1902)}

Cochabamba: Parotani (Boulenger, 1902c). 
Cnemidophorus lacertoides Duméril \& Bibron, 1839 Cochabamba: Arani, UMMZ. Santa Cruz: El Fuerte (Dirksen et al., 1995). Santiago, FMNH.

\section{Cnemidophorus ocellifer (Spix, 1825)}

Santa Cruz: Cerro Colorado; La Madre; Tierras Nuevas (Gonzáles, 1998). Tarija: Caiza (Peracca, 1897). Villa Montes (Hellmich, 1960).

\section{Dracaena paraguayensis Amaral, 1950}

Santa Cruz: El Refugio (Harvey, 1995).

\section{Kentropyx altamazonica Cope, 1876}

Cochabamba: Charuplaya; Yungas (Gallagher \& Dixon, 1992). Puerto San Mateo (Avila-Pires, 1995). $50 \mathrm{~km} \mathrm{NW}$ of Villa Tunari, Yungas de Cochabamba, UMMZ. La Paz: Huachi (Gallagher \& Dixon, 1992). San Fermín (Avila-Pires, 1995). Santa Cruz: Parque Nacional Amboró (Lavilla et al., 1996). Río Cheyo, CET.

\section{Kentropyx calcarata Spix, 1825}

Beni: Camiaco; 4 km W of Puerto Cap. Vázquez; Puerto Siles; Río Blanco (4-5 km from confluence with Río Iténez), AMNH. Cochabamba: Río Ichilo, ca. 54 km S Boca Chapare, AMNH. La Paz: Misiones Mosetenes (Boulenger, 1898). Santa Cruz: Puerto Almacén (De la Riva, 1993). Puerto Suárez (Montero et al., 1995). Campamento Las Torres (Scrocchi \& Gonzáles, 1996). Cupesí; La Madre (Gonzáles, 1998). Piso Firme, AMNH. Tarija: Villa Montes (Gallagher \& Dixon, 1992).

\section{Kentropyx pelviceps Cope, 1868}

Pando: San Juan del Nuevo Mundo, USNM.

\section{Kentropyx vanzoi Gallagher \& Dixon, 1980}

Santa Cruz: Serranía de Huanchaca (Montero et al., 1995). Flor de Oro; Meseta de Caparuch (Scrocchi \& Gonzáles, 1996).

\section{Kentropyx viridistriga Boulenger, 1894}

Santa Cruz: Río Surutú (Gallagher \& Dixon, 1992). Buena Vista (Lavilla et al., 1996).

\section{Teius teyou (Daudin, 1802)}

Chuquisaca: $30 \mathrm{~km}$ SE of Carandayti, LACM. Santa Cruz: Santa Cruz de la Sierra (Duméril \& Bibron, 1839). San José de Chiquitos (Müller, 1928). El Carmen; Roboré (Gans, 1960). Los Troncos (Hellmich, 1960). Perforación (De la Riva et al., 1992). Curuyuqui (Emmons, 1993). Cupesí; La Madre; Tierras Nuevas; Yapiroa (Gonzáles, 1998). Boyuibi, LACM. Lomas de Arena, MNK. Camiri, ZFMK. Tarija: San Francisco (Peracca, 1897). Fortín Magariños (Mertens, 1929). Capirenda, LACM.

Tupinambis merianae (Duméril \& Bibron, 1839) Santa Cruz: Parque Nacional Noel Kempff Mercado (Harvey et al., 1998 ).

\section{Tupinambis rufescens (Günther, 1871)}

Beni: Lago Victoria (Presch, 1973). San Joaquín, FMNH. Santa Cruz: Puerto Suárez (Presch, 1973). Aguaraigua; Cupesí; La Madre; Tierras Nuevas (Gonzáles, 1998). Santiago, FMNH. Santa Cruz, USNM. Tarija: Caiza; San Francisco (Peracca, 1897).

\section{Tupinambis teguixin (Linnaeus, 1758)}

Beni: Costa Márquez, Río Iténez; Santa Rosa, Río Mamoré (Presch, 1973). Tumi Chucua (Fugler, 1983). Estancia El Porvenir (De la Riva et al., 1992). Guayaramerín; Puerto Cap. Vázquez; Puerto Versalles; confluence of Río Blanco and Río Iténez; Río Mamoré (133ㅗ' S, $\left.12^{\circ} 26^{\prime} \mathrm{S}\right)$; 10 km W San Pedro; Santa Fé; Trinidad, AMNH. San Joaquín, FMNH. Cochabamba: ca. $54 \mathrm{~km} \mathrm{~S}$ of Boca Chapare, AMNH. Pando: Reserva Nacional Amazónica Manuripi-Heath (Aparicio, 1992). Santa Cruz: El Carmen (Gans, 1960). San José de Chiquitos (Hellmich, 1960). Puerto Almacén (De la Riva, 1993). Inselberg (Dirksen, 1995). El Refugio (Harvey, 1995). Parque Nacional Amboró (Lavilla et al., 1996). Cupesí; La Madre (Gonzáles, 1998). Abapó; Charagua; San Antonio de Parapetí, AMNH. Buena Vista, UMMZ.

\section{TROPIDURIDAE}

\section{Liolaemus alticolor Barbour, 1909}

Cochabamba: Province Arque (Ibisch \& Böhme, 1993). $36 \mathrm{~km} \mathrm{~W}$ of Challa; $38 \mathrm{~km} \mathrm{~W}$ of Challa, KU. La Paz: Tihuanacu (Barbour, 1909). Cheripa (Rendahl, 1937). Huatajata; Sorata (Hellmich, 1961). La Paz; Patamanta; Península de Taraco, Huacullani (Fugler, 1989). Achocalla; Alto Següencoma; Collana; road to Collana from Lipari; La Cumbre; Huajchilla; upper Río Irpavi near Karpani; Jupapina; Río Kaluyo; Lipari; Llojeta; Mallasa; Río Ovejuyo; hill bordering the road between Avda. Poeta and Villa Holguín (Baudoin \& Pacheco, 1991). Patacamaya (De la Riva et al., 1992). Huaraco (Aparicio, 1993). $35 \mathrm{~km}$ on the road from La Paz to Oruro (Dirksen, 1995). Esperanza, Province Pacajes, FMNH. $13 \mathrm{~km}$ E of El Tambillo, Río Pallina, KU. $10 \mathrm{~km}$ E of Achacachi, Hacienda Collana, halfway between Ayo-Ayo and Calamarca, UMMZ. Kallutaca, ca. $15 \mathrm{~km} \mathrm{~N}$ of La Paz, USNM. Oruro: Oruro; $15 \mathrm{~km} \mathrm{NE}$ of Oruro; Playa Verde, AMNH. 17 km SE of Caracollo, KU. Potosí: Dpto. Potosí, AMNH. Villazón, MVZ.

\section{Liolaemus cranwelli (Donoso-Barros, 1973)}

Santa Cruz: Nueva Moka (Donoso-Barros, 1973).

Liolaemus dorbignyi Koslowsky, 1898

Potosí: Campamento Khastor (Laurent, 1995).

Liolaemus fittkaui Laurent, 1986

Cochabamba: "Región de Cochabamba"; Tiraque (Laurent, 1986).

Liolaemus forsteri Laurent, 1982

La Paz: Chacaltaya, 4700 m (Laurent, 1982). 
Liolaemus islugensis Ortiz \& Marquet, 1987

Potosí: Laguna Colorada; $40 \mathrm{~km} \mathrm{~S}$ of Laguna Colorada; Salar de Chalvirí (Laurent, 1995).

\section{Liolaemus jamesi (Boulenger, 1891)}

Potosí: Laguna Colorada (Laurent, 1995).

\section{Liolaemus mocquardi Pellegrin, 1909}

La Paz:Tihuanacu (Pellegrin, 1909). Alto Achachicala, Río Caluyo; La Paz; Province Aroma, Huaraco; Reserva Ulla Ulla, Cordillera de Apolobamba, Huacullani (Fugler, 1989). Patacamaya (De la Riva et al., 1992). Oruro: Crequi (Pellegrin, 1909). $203 \mathrm{~km}$ from La Paz to Oruro along highway, USNM. Potosí: E of Potosí (Fugler, 1989).

\section{Liolaemus orientalis Müller, 1924}

Chuquisaca: Paso de Macho Cruz (Fugler, 1989). Potosí: Paso de Macho Cruz; Province near Chichas (Fugler, 1989). Laguna Colorada (Laurent, 1995). Portugalete, AMNH. Tarija: Río Pilcomayo, between Tarija and San Francisco (Müller, 1923).

\section{Liolaemus ornatus Koslowsky, 1898}

La Paz: Tihuanacu (Pellegrin, 1909). Murillo, $6 \mathrm{~km} \mathrm{~W}$ of Patacamaya, UMMZ. Oruro: Lago Poopó (Fugler, 1989). Crequi (Pellegrin, 1909). $8 \mathrm{~km} \mathrm{~S}$ of Eucaliptos, UMMZ. Potosí: Uyuni, FMNH. Villazón, MVZ.

\section{Liolaemus pantherinus Pellegrin, 1909} 1909).

Cochabamba: Tiraque, UMMZ. Oruro: Crequi (Pellegrin,

Liolaemus pulcher Pellegrin, 1909

La Paz: Tihuanacu (Pellegrin, 1909). Oruro: Crequi; Senechal (Pellegrin, 1909). $15 \mathrm{~km} \mathrm{NE}$ of Oruro, AMNH.

\section{Liolaemus schmidti (Marx, 1960) \\ Potosí: Julaca, FMNH.}

\section{Liolaemus signifer (Duméril \& Bibron, 1837)}

Cochabamba: Province Arque (Ibisch \& Böhme, 1993). $5.5 \mathrm{~km}$ Wof Challa; $36 \mathrm{~km} \mathrm{~W}$ of Challa; $38 \mathrm{~km} \mathrm{~W}$ of Challa, KU. La Paz: Chilalaya; Lago Titicaca (Boettger, 1891). Querqueta; San José at the lake Titicaca (Rendahl, 1937). Huatajata; La Paz; Pucarani; Sorata (Hellmich, 1962). Alto Achachicala, Río Caluyo; Province Murillo; Province Pacajes; km 74 by road between Sorata and La Paz (Fugler, 1989). Road to Chacaltaya; Jacha Toloko; La Cumbre; upper Río Kaluyo; upper Río Ovejuyo (Baudoin \& Pacheco, 1991). Ulla-Ulla (De la Riva et al., 1992). Huaraco (Aparicio, 1993). Road between Caracoles and Quime; Cordillera Tres Cruces; road between La Paz and Tiahuanaco; La Cumbre; $130 \mathrm{~km} \mathrm{NE}$ of La Paz; $35 \mathrm{mi}$ NW of La Paz; Potone; Tiahuanaco; $7 \mathrm{~km}$ E of Ulla Ulla, AMNH. Esperanza, Province Pacajes, FMNH. Caupolicán, Hacienda Corpaputu, 10 km E of Achacachi; Patacamaya, UMMZ. Oruro: Crequi; Senechal (Pellegrin, 1909). Eucaliptos, NW of Lake Poopo
(Rendahl, 1937). Altiplano; Oruro; $5 \mathrm{~km} \mathrm{NE}$ of Oruro; $15 \mathrm{~km}$ NE of Oruro; $60 \mathrm{~km}$ NE of Oruro, AMNH. Hacienda Huancaroma; $8 \mathrm{~km} \mathrm{~S}$ of Eucaliptos, UMMZ. Potosí: Potosí (Fugler, 1989). Chocaya, ANSP. $30 \mathrm{mi} \mathrm{N}$ of Potosí, CAS. 7 km S of Potosí, KU.

\section{Liolaemus simonsii Boulenger, 1902}

Chuquisaca: Sucre, AMNH. Oruro: Challapata (Boulenger, 1902c). Eucaliptos, N of Lago Poopo (Rendahl, 1937). Playa Verde, AMNH. Potosí: Potosí; Uyuni (Boulenger, 1902c). Near Chichas (Fugler, 1989). Tarija: between Tarija and San Francisco (Müller, 1924).

\section{Liolaemus variegatus Laurent, 1984}

Cochabamba: Tiraque (Laurent, 1984). Cliza, KU. Arani; Pocona; Tiraque; Totora, UMMZ.

\section{Stenocercus aculeatus (O’Shaughnessy, 1879)}

Beni: Tumi Chucua (Fugler, 1983).

\section{Stenocercus caducus (Cope, 1862)}

Beni: Reyes (Boulenger, 1898). Rancho San Marcos, in the confluence of the Isiboro and Ichoa rivers (Fugler, 1989). Rurrenabaque; $10 \mathrm{~km}$ E of San Antonio, AMNH. Chuquisaca: El Palmar (Harvey, 1997). Cochabamba: 6.5 $\mathrm{km} N$ of Chipiriri, KU. La Paz: Barraca, Río Madidi; Chulumani; Coroico; Misiones Mosetenes (Boulenger, 1898). Tumupassa, AMNH. Santa Cruz: Buena Vista; Santa Cruz de la Sierra (Burt \& Burt, 1931). San José de Chiquitos (Hellmich, 1960). Perseverancia (Hoogmoed \& Vaca, 1990). Puerto Almacén (De la Riva, 1993). $35 \mathrm{~km}$ on the road from St. Rosa de la Roca to Piso Firme (Dirksen, 1995). El Refugio (Harvey, 1995). Campamento Ichilo; Caranda; La Guitarra; Parque Nacional Amboró (Lavilla et al., 1996). Cupesí; La Madre; Tierras Nuevas; Yapiroa (Gonzáles, 1998). $23 \mathrm{~km} \mathrm{~N}$ of Boca Chapare, AMNH. Santiago, FMNH. Tarija: Aguairenda; San Francisco (Peracca, 1897).

Stenocercus marmoratus (Duméril \& Bibron, 1837)

Chuquisaca: Pampa Ruíz (D’Orbigny, 1847). El Palmar (Harvey, 1997). Cochabamba: Cochabamba (Werner, 1910). Province Arque (Ibisch \& Böhme, 1993). Arani; Pocona; Tiraque; Totora, UMMZ. Santa Cruz: El Fuerte (Dirksen et al., 1995). Tarija: Entre Ríos (Harvey, 1997).

Stenocercus roseiventris Duméril \& Bibron, 1837

Beni: Province Beni (Werner, 1913). Riberalta, UMMZ. Chuquisaca: Sucre, FMNH. Cochabamba: Charuplaya (AvilaPires, 1995). Yungas de Cochabamba, MVZ. La Paz: Cordillera de Irupana (D’Orbigny, 1847). Misiones Mosetenes (Boulenger, 1898). 10 km NWof Mapiri, UMMZ. Santa Cruz: Province Sara (Griffin, 1917). Buena Vista; Campamento Ichilo; La Siberia; Parque Nacional Amboró (Lavilla et al., 1996). Río Surutú, UMMZ.

Stenocercus variabilis Boulenger, 1901

La Paz: Palca (Boulenger, 1901). 
Tropidurus callathelys Harvey \& Gutberlet, 1998

Santa Cruz: Northern slope of the Serranía de Huanchaca, near Lago Caimán (Harvey \& Gutberlet, 1998).

Tropidurus chromatops Harvey \& Gutberlet, 1998

Santa Cruz: Inselberg (Dirksen, 1995). Campamento Las Torres; $10 \mathrm{~km}$ E of Florida on the road to Los Fierros; Northern slope of the Serranía de Huanchaca (Harvey \& Gutberlet, 1998)

\section{Tropidurus etheridgei Cei, 1982}

Beni: $23 \mathrm{~km} \mathrm{~W}$ of San Javier; $10 \mathrm{~km} \mathrm{~W}$ of San Pedro; Trinidad, AMNH. Chuquisaca: $30 \mathrm{~km} \mathrm{SE}$ of Carandayti, LACM. Cochabamba: N of Río Mizque; Peña Colorada; E of Villa Granado (Rodrigues, 1987). $20 \mathrm{~km} \mathrm{E} \mathrm{of} \mathrm{Cochabamba} \mathrm{(De}$ la Riva et al., 1992). $15 \mathrm{~km} \mathrm{~S}$ of Quiroga, KU; Parotani; $3 \mathrm{~km}$ N of Mizque; Viña Perdida Farm, UMMZ. La Paz: $1.6 \mathrm{~km} \mathrm{~W}$ of Puerto Linares (Fugler, 1984). Santa Cruz: El Carmen; Naranjos; El Portón (Gans, 1960). Roboré; San Lorenzo (Rodrigues, 1987). $69 \mathrm{~km}$ from Samaipata on the road Samaipata - Cochabamba (Dirksen, 1995). Mataral (Montero et al., 1995). Aguaraigua; Cerro Colorado; La Madre; Tierras Nuevas; Yapiroa (Gonzáles, 1998). Charagua; La Brecha; San Antonio de Parapetí, AMNH. Lake Gahibia-Mirim; Santiago, FMNH. Comarapa, UMMZ. Tarija: Aguairenda; Caiza; San Francisco (Peracca, 1897). Capirenda, LACM. Villa Montes, ZFMK.

\section{Tropidurus melanopleurus Boulenger, 1902}

La Paz: Tamampoya (Boulenger, 1902c). $25.4 \mathrm{~km} \mathrm{~S}$ of Caranavi; $29 \mathrm{~km} \mathrm{~S}$ of Caranavi; $64.3 \mathrm{~km} \mathrm{~S}$ of Caranavi, KU. 20 $\mathrm{km}$ from Caranavi to Santa Ana de Huachi; $1 \mathrm{~km} \mathrm{~S}$ of San Buenaventura; $25 \mathrm{~km} \mathrm{~S}$ of Yolosa, UMMZ. Zongo Valley, at Jarca Electric Plant, USNM. Chuquisaca: El Palmar (Harvey, 1997). Santa Cruz: Cerro Hosane (Fugler, 1989). Samaipata (Pérez-Mellado \& De la Riva, 1993). El Fuerte (Dirksen et al., 1995). La Guitarra; Parque Nacional Amboró; Río Cheyo (Lavilla et al., 1996). $6 \mathrm{~km} \mathrm{~W}$ of Charagua; San Antonio de Parapetí, AMNH. Tarija: Río Pilcomayo, between Tarija and San Francisco (Müller, 1923). Tarairi, N of Villa Montes (Mertens, 1929). Villa Montes (Fugler, 1989). 45 km W of Río Seco; $20 \mathrm{~km} \mathrm{~N}$ of Villa Montes, ZFMK.

\section{Tropidurus plica (Linnaeus, 1758)}

Pando: Abuná (Schmidt \& Inger, 1951). Ca. $12 \mathrm{~km}$ by road $\mathrm{S}$ of Cobija and $8 \mathrm{~km}$ from Mucden (Fugler, 1988c).

\section{Tropidurus spinulosus (Cope, 1862)}

Beni: Trinidad, AMNH. Chuquisaca: $30 \mathrm{~km}$ SE of Carandayti, LACM. Santa Cruz: Puerto Suárez (Griffin, 1917). $1 \mathrm{~km} \mathrm{~N}$ of El Carmen; El Carmen; San José de Chiquitos (Gans, 1960). Mountains of Chiquitos (Hellmich, 1960). Cerro Colorado (De la Riva et al., 1992). Aguaraigua; Cupesí; La Madre; Tierras Nuevas; Yapiroa (Gonzáles, 1998). Finca de Milanos (Harvey \& Gutberlet, 1998). La Brecha; San Antonio de Parapetí, AMNH. Tarija: San Francisco (Peracca, 1897). $12 \mathrm{~km} \mathrm{SW}$ of Capirenda, LACM. Taringuiti, UMMZ.

\section{Tropidurus umbra (Linnaeus, 1758)}

Beni: Guayaramerín (Etheridge, 1970). Tumi Chucua (Fugler, 1983). La Paz: San Fermín (Andersson, 1918). Pando: Puesto Abuná, USNM. Santa Cruz: Los Fierros; Florida (De la Riva et al., 1992). Flor de Oro; Lago Caimán (Harvey \& Gutberlet, 1998). Parque Nacional Amboró (Lavilla et al., 1996).

Tropidurus xanthochilus Harvey \& Gutberlet, 1998 Santa Cruz: El Refugio (Harvey, 1995).

Uranoscodon superciliosus (Linnaeus, 1758)

Beni: Guayaramerín, AMNH.

\section{Conclusions}

The lizards and amphisbaenians of Bolivia consist of ten families, 39 genera and 102 species (13 Amphisbaenidae, 3 Anguidae, 9 Gekkonidae, 15 Gymnophthalmidae, 2 Hoplocercidae, 1 Iguanidae, 8 Polychrotidae, 7 Scincidae, 14 Teeidae and 30 Tropiduridae). We add 24 species to the provisional checklist compiled by Fugler (1989), of which three are here reported for the first time: Kentropyx pelviceps, Liolaemus schmidti, and Uranoscodon superciliosus. This addition represents an increment of $29 \%$ in the saurian and amphisbaenian diversity of the country. Four species listed in Fugler (1989) do not occur in Bolivia or are synonyms: Tropidurus hispidus occurs in the Northeast of South America, Liolaemus multifor mis is a synonym of Liolaemus signifer, Homonota horrida of Homonota fasciata and Teius cyanogas ter of Teius teyou. It is surprising that Fugler (1989) did not mention the genus Kentropyx in Bolivia.

Nine species are endemic to Bolivia: Amphisbaena bolivica, Amphisbaena cegei, Neusticurus ocellatus, Mabuya cochabambae, Liolaemus fittkaui, Liolaemus forsteri, Liolaemus islugensis, Liolaemus variegatus and Stenocercus marmoratus.

Many new reptilian species were described in the last 10-20 years in South America, including Bolivia, where large areas still remain to be surveyed. It is realistic to assume that there are many more undescribed Bolivian species awaiting to be discovered, especially in the families Amphisbaenidae, Gymnophthalmidae and Tropiduridae. As a result, the numbers given above will increase in the future. New findings must be expected near the borders of the Amazonian Brazil, the Andean slopes of Peru and the highlands near Argentina and Chile. 


\section{ACKNOWLEDGMENTS}

This research has been possible by the help of many persons and by the splendid collaboration with the museum staffs. We wish to thank all of them and, in particular, James Aparicio (CBF), Robert L. Besy (LACM), Wolfgang Böhme (ZFMK), Robert Drewes (CAS), William Duellman (KU), Richard Etheridge (SDSU), Linda S. Ford (AMNH), Lucindo Gonzáles (MNK), Rainer Günther (ZMB), Jakob Hallermann (ZMH), Monique Halloy (FML), Marinus S. Hoogmoed (RMNH), Pierre L. Ibisch (FAN), Arnold G. Kluge (UMMZ), Jörn Köhler (ZFMK), Stefan Lötters (ZFMK), Charles W. Myers (AMNH), Alan Resetar (FMNH), Greg Schneider (UMMZ), Gustavo Scrocchi (FML), John Simmons (KU), Barbara R. Stein (MVZ), and Harold Voris (FMNH). D. Frost and J. Wiens helped with some particularly problematic species. This paper is funded by project DGES PB97-1147, Spanish Ministry of Education and Culture (I. De la Riva, principal investigator).

\section{References on Bolivian saurians and amphisbaenians}

Amaral, A., 1950. Two new South American lizards. Copeia, 1950(4): 281-284.

Andersson, L. G., 1914. A new Telmatobius and new teiid lizards from South America. Ark. Zool., 9(3): 112.

Andersson, L. G., 1918. New lizards from South America. Ark. Zool., 11(6): 1-10.

ApAricio, J., 1992. Herpetofauna. In: H. Salm \& M. Marconi (eds.), Reserva Nacional Amazónica Manuripi-Heath. Programa de reestructuración (Fase II), PL-480, LIDEMA, CORDEPANDO. La Paz: 113-119.

APARICIO, J., 1993. Herpetofauna de Huaraco, un ecosistema andino en el altiplano central de Bolivia. Ecol. Bolivia Doc. Zool., 4: 1-38.

Aquino, A. L., Scott, N. \& Motte, M., 1996. Colecciones de flora y fauna del Museo Nacional de Historia Natural del Paraguay (1996): Lista de anfibios y rep tiles del Museo de Historia Natural del Paraguay (Marzo, 1980 - Setiembre, 1995). Museo Nacional de Historia Natural del Paraguay. Asunción: 332-400.

Avila-Pires, T. C. S., 1995. Lizards of Brazilian Amazonia. Zool. Verh., 299: 1-706.

BArbour, T., 1909. Some new South American coldblooded vertebrates. Proc. New England Zool. Club, 4: 47-52.

Barbour, T., 1934. The Anoles. II. The mainland species from Mexico southward. Bull. Mus. Comp. Zool., 77(4): 121-155.

Baudoin, M. \& Pacheco, L., 1991. Reptiles. In: Baudoin, M. \& E. Forno (eds.): Historia Natural de un valle en los Andes: La Paz. Instituto de Ecología, UMSA. La Paz: 421-452.

Bocourt, M.-F., 1870-1909. In: Duméril, Bocourt \& Mocquard. Recherches zoologiques pour servir a l'histoire de la faune de l'Amérique. 1873. Part III. Etudes sur les reptiles et les batraciens. 2ièmé livre. Mission scientifique au Mexique et dans 1'Amerique Centrale.

Boettger, O., 1885. Liste von Reptilien und Batrachian aus Paraguay. Zeitschr. Naturwiss., 58: 213-248.

Boettger, O., 1891. Reptilien und Batrachian aus Bolivien. Zool. Anz., 14: 343-347.

Boulenger, G. A. 1883. Description of a new species of lizard of the genus Enyalius. Proc. Zool. Soc. London, 1883(1): 46.

Boulenger, G. A., 1885. Catalogue of lizards in the British Museum (Natural History). 2nd Edition. London. Vol. 1: 1-436, pls 1-32; Vol. 2: 1-497, pls 124.

Boulenger, G. A., 1891. Description of a new lizard of the genus Ctenoblepharis, from Chile. Proc. Zool. London, 1891: 3.

Boulenger, G. A., 1894. List of reptiles and batrachians collected by Dr. Bohls near Asunción, Paraguay. Ann. Mag. Nat. Hist., (13), 6: 342-348.

Boulenger, G. A., 1898. Alist of reptiles and batrachians collected by the late Professor L. Balzan in Bolivia. Ann. Mus. Civ. Storia Nat. Genova, (2), 19: 1-6.

Boulenger, G. A., 1901. Further descriptions of new reptiles collected by Mr. P. O. Simmons in Peru and Bolivia. Ann. Mag. Nat. Hist., (7), 7: 546-549.

Boulenger, G. A., 1902a. Descriptions of new fishes and reptiles discovered by Dr. F. Silvestre in South America. Ann. Mag. Nat. Hist., (9), 7: 284-288.

Boulenger, G. A., 1902b. List of fishes, batrachians and reptiles collected by the late Mr. P. O. Simmons in the provinces of Mendoza and Cordoba, Argentina. Ann. Mag. Nat. Hist., (9), 7: 336-339.

Boulenger, G. A., 1902c. Descriptions of new batrachians and reptiles from the Andes of Peru and Bolivia. Ann. Mag. Nat. Hist., (10), 7: 394-402.

Boulenger, G. A., 1907. Descriptions of new lizards in the British Museum. Ann. Mag. Nat. Hist., 19(7): 486-489.

Boulenger, G. A., 1908. Descriptions of new South American reptiles. Ann. Mag. Nat. Hist., (1), 8: 111115.

Boulenger, G. A., 1912. Descriptions of new reptiles from the Andes of South America, preserved in the British Museum. Ann. Mag. Nat. Hist., (8), 10(58): 420-424.

Burmeister, G., 1861. Reise durch die La Plata Staaten. Vol. II. Halle. 538 pp.

Burt, C. E. \& Burt, M. D., 1930. The South American lizards in the collection of the United States National Museum. Proc. U. S. Natl. Mus., 78(6): 1-51.

Burt, C. E. \& BurT, M. D., 1931. South American lizards in the collection of the American Museum of 
Natural History. Bull. Amer. Mus. Nat. Hist., 61: 227 395.

CARrillo De EspinozA, N \& ICOChEA, J., 1995. Lista taxónomica preliminar de los reptiles vivientes del Perú. Publ. Mus. Hist. Nat. UNMSM, 49: 1-27.

CEI, J. M., 1982. A new species of Tropidurus (Sauria, Iguanidae) from the arid Chacoan and western regions of Argentina. Occ. Pap. Mus. Nat. Hist. Univ. Kansas, 97: 1-10.

CEI, J. M., 1993. Reptiles del noroeste, nordeste y este de la Argentina. Herpetoafauna de las selvas subtropicales, Puna y Pampas. Mus. Reg. Sci. Nat. Torino, Monog., 14. 949 pp.

CoPE, E. D., 1861. Some remarks defining the following species of Reptilia Squamata. Proc. Acad. Nat. Sci. Philadelphia, 13: 75-77.

Cope, E. D., 1862a. Catalogue of the reptiles obtained during the explorations of the Paraná, Paraguay, Bermejo and Uruguay Rivers by Captain Thomas L. Page, U. S. N. and those procured by Lt. N. Michler, U. S. Top. Eng. Commander of the expedition conducting the survey of the Atrato River. Proc. Acad. Nat. Sci. Philadelphia, 14: 346-359.

Cope, E. D., 1862b. Contributions to neotropical saurology. Proc. Acad. Nat. Sci. Philadelphia, 14: 176188.

Cope, E. D., 1868. An examination of the Reptilia and Batrachia obtained by the Orton expedition to Ecuador and the upper Amazon with notes on other species. Proc. Acad. Nat. Sci. Philadelphia, 20: 96119.

Cope, E. D., 1876. Report on the reptiles brought by Professor James Orton from the middle and upper Amazon and western Peru. J. Acad. Nat. Sci. Philadelphia, (2), 8(1875): 159-188.

Cope, E. D., 1885. Twelfth contribution to the herpetology of tropical America. Proc. Amer. Philos. Soc., 22: 167-194.

DAUDIN, F. M., 1800-1804. Histoire naturelle générale et particulière des reptiles. 8 vols. Paris. Vol. 3: 452 pp.; Vol. 4: 397 pp.

DE LA Riva, I., 1993. Ecología de una comunidad neo tropical de anfibios durante la estación lluviosa. $\mathrm{PhD}$ Dissertation. Universidad Complutense, Madrid. 365 pp.

De la Riva, I., Castroviejo, J. \& Cabot, J., 1992. Pseustes sulphureus (Wagler, 1824) (Serpentes: Colubridae) nueva especie para Bolivia, y datos sobre la herpetofauna boliviana. Acta zool. Lilloana, 41: $215-218$.

Dirksen, L., 1995. Zur Reptilienfauna Boliviens unter spezieller Berücksichtigung taxonomischer und zoo geographischer Aspekte. Diplomarbeit der Rheinischen Friedrich-Wilhelm-Universität Bonn (ZFMK). 172 pp.
Dirksen, L., IBISCH, P. L., KÖHLER, J. \& BÖHME, W., 1995. Zur Herpetofauna der semihumiden SamaipataRegion, Bolivien. II Reptilien. Herpetofauna, 17(99): 15-28.

Dixon, J. R., 1973. A systematic review of the teiid lizards, genus Bachia, with remarks on Heterodactylus and Anotosaura. Univ. Kansas Mus. Nat. Hist. Misc. Publ., 57: 1-47.

Dixon, J. R., 1974. Systematic review of the microteiid genus Iphisa. Herpetologica, 30: 133-139.

Donoso-Barros, R., 1973. Un nuevo saurio de Bolivia (Lacertilia, Iguanidae). Neotrópica, 19(60): 132-134.

D’Orbigny, A., 1837. In: Duméril, A. M. C., Bibron, G. \& Duméril, A. H. A., 1834-1854. Herpétologie géné rale ou histoire naturelle complète des reptiles. Vol. 4. Roret. Paris. 517 pp.

D'Orbigny, A., 1847. Voyage dans l'Amerique Meridionale. Tome V, 1ère partie. Reptiles. Chez Bertrand. Paris.

Duméril, A. M. C. \& BiBron, G., 1836. Herpétologie générale ou histoire naturelle complète des reptiles. Vol. 3. Roret. Paris. 517 pp., pl. 1-36.

Duméril, A. M. C. \& BiBron, G., 1837. Herpétologie générale ou histoire naturelle complète des reptiles. Vol. 4. Roret. Paris. 571 pp., pl. 37-48.

Duméril, A. M. C. \& BiBron. G., 1839. Herpétologie générale ou histoire naturelle complète des reptiles. Vol. 5. Roret. Paris. 854 pp., pl. 49-58.

DunN, E. R., 1935. Notes on American mabuyas. Proc. Acad. Nat. Sci. Philadelphia, 87: 533-557.

EMmONS, L. H., 1991. Observations on the herpetofauna. In: Parker, T. \& Bailey, B. (eds.): A biological assess ment of the Alto Madidi region and adjacent areas of Bolivia. RAP Working Papers 1: 74.

Emmons, L. H., 1993. Reptile and amphibian species recorded in the Curuyuqui Chaco Forest. In: Parker, T., Foster, R. B., Emmons, L. \& Bailey, B. (eds.): The lowland dry forests of Santa Cruz, Bolivia: a global conservation priority. RAP Working Papers 4: 104.

Etheridge, R., 1970. A review of the South American iguanid lizard genus Plica. Bull. Brit. Mus. Nat. Hist. (Zool.), 19(7): 237-256.

Etheridge, R. \& Williams, E. E., 1991. A review of the South American lizards genera Urostrophus and Anisolepis (Squamata: Iguania: Polychridae). Bull. Mus. Comp. Zool., 152(5): 317-361.

FITZINGER,L., 1843. Systema Reptilium, Fasciculus primus, Amblyglossae. Braumüller et Seidel. Wien. 106 pp.

Frost, D. R. \& Etheridge, R., 1989. A phylogenetic analysis and taxanomy of iguanian lizards (Reptilia: Squamata). Univ. Kansas, Mus. Nat. Hist., Misc. Publ., 81: 1-65.

Fugler,C. M., 1983. Lista preliminar de los anfibios y reptiles de Tumi Chucua. Mus. Nac. Hist. Nat. (Bolivia) Comunicación, 2: 4-11. 
Fugler, C. M., 1984. Tercera contribución a la fauna herpetológica del Oriente boliviano. Ecol. Bolivia, 5: 63-72.

FugLER, C. M., 1985. Adiciones y correcciones a la lista preliminar de la herpetofauna de Tumi Chucua, province Vaca Díez, Departamento de Beni. Cuad. Acad. Ciec. Bolivia, Zool., 3: 17-18.

Fugler, C. M., 1986. La estructura de una comunidad herpetológica en las selvas benianas en la estación de sequía. Ecol. Bolivia, 8: 1-20.

Fugler, C. M., 1988a. La existencia de Hemidactylus mabouia (Sauria: Gekkonidae) en las selvas bolivianas. Mus. Nat. Hist. Nac. (Bolivia) Comunicación, 6: 17.

Fugler, C. M., 1988b. Anolis punctatus (Daudin) (Reptilia: Iguanidae), un nuevo saurio registrado en Bolivia. Mus. Nat. Hist. Nac. (Bolivia) Comunicación, 8: $5-6$

Fugler, C. M., 1988c. Plica plica (Linnaeus) (Sauria: Iguanidae), adición a la herpetofauna boliviana. Mus. Nat. Hist. Nac. (Bolivia) Comunicación, 8: 3-4.

Fugler, C. M., 1988d. Consideraciones sobre la herpetofauna de las selvas tropicales bolivianas. Mus. Nac. Hist. Nat. (Bolivia) Comunicación, 6: 3-12.

Fugler, C. M., 1989. Lista de Saurios. Ecol. Bolivia, 13: 57-75.

Gallagher, D. S. \& Dixon, J. R., 1980. A new lizard (Sauria: Teiidae: Kentropyx) from Brazil. Copeia, 1980(4): 616-620.

Gallagher, D. S. \& Dixon, J. R., 1992. Taxonomic revision of the South American lizard genus Kentropyx Spix (Sauria: Teiidae). Boll. Mus. reg. Sci. nat. Hist. Torino, 10(1): 125-171.

GALlardo, J. M., 1966. Las especies argentinas del género Ophiodes Wagler. Rev. Mus. Arg. Cienc. Nat. Bernardino Rivadavia (Cienc. Zool.), 9(6): 123-146.

GANS, C., 1960. Notes on a herpetological collecting trip through the southeastern lowlands of Bolivia. Ann. Carnegie Mus., 13: 288-314.

GANs, C., 1964. New records of Amphisbaena silvestrii Boulenger, and the description of new two-pored species from the Northern Chaco. Copeia, 1964(3): $553-561$

GANS, C., 1965. Notes on amphisbaenids (Amphisbaenia, Reptilia). A redescription and discussion of Amphisbaena angustifrons Cope and Amphisbaena camura Cope of southern South America. Amer. Mus. Nov., 2225: 1-32.

GANS, C., 1967. Redescription of Amphisbaena slateri Boulenger, with comments on its range extension into Bolivia. Herpetologica, 23(3): 223-227.

GonzÁLes, L., 1998. La herpetofauna del Izozog. Ecol. Bolivia, 31: 45-51.

GraY, J. E., 1831. A synopsis of the species of the class Reptilia. In: Griffith, E. \& Pidgeon, E. (eds.). The animal kingdom arranged in conformity with its organization, by the Baron Cuvier, member of the Institute of France, with additional descriptions of all the species hitherto named, and of many of before noticed. Volume the ninth. The class Reptilia arranged by the Baron Cuvier, with specific descrip tions. Whittaker Treacher, and Co. London: 1-110 (appendix).

GraY, J. E., 1851. Description of a new genus and family of cyclosaurian lizards, from Pará. Proc. Zool. Soc. London, 1851: 38-39.

GRIFFIN, L. E., 1917. A list of South American lizards of the Carnegie Museum, with descriptions of four new species. Ann. Carnegie Mus., 11: 304-320.

GuiCHENOT, A., 1855. Animaux nouveaux ou rare recui llis pendant l'expédition dans les parties centrales de l'Amerique du Sud, de Río de Janeiro à Lima, et de Lima au Pará; executée par l'ordre du gouvernment français pendant les années 1843 a 1847, sous la direction du Compte Francis de Castelnau. Vol. II. Reptiles. Paris. Bertrand. 96 pp., 18 pls.

GüNTHER, A., 1871. Description of a new species of Tejus (Tejus rufescens) from Mendoza. Proc. Zool. Soc. London: 541-543.

HARris, D. N., 1994. Review of the teiid lizard genus Ptychoglossus. Herp. Monogr., 8: 226-275.

HARveY, M. B., 1995. A preliminary list of the reptiles and amphibians of the El Refugio biological reserve. In: Forsyth, A. (ed.). A report on aspects of biodiver sity and conservation potential in El Refugio. Conservation International. Washington: 21-26.

Harvey, M. B., 1997. Reptiles and amphibians from the vicinity of El Palmar in the Andes of Chuquisaca. In: Schulenberg, T. \& Awbrey, K. (eds.). A rapid assess ment of the humid forests of south centra Chuquisaca, Bolivia. Conservation International, Washington. RAP Working Papers 8: 33-36, 83-84.

HARVEY, M. B., 1998. Reptiles and amphibians of Parque Nacional Noel Kempff Mercado. In: Killeen, T. J. \& T. S. Schulenberg (eds). A biological assess ment of Parque Nacional Noel Kempff Mercado, Bolivia. Conservation International Washington. RAPWorking Papers 10: 144-166.

Harvey, M. B., Aparicio, J., Cortez, C., GonzÁles, L., Guerra, J. F., Montaño, M. E. \& Pérez, M. E., 1998. Reptile and amphibian species of Parque Nacional Noel Kempff Mercado. In: Killeen, T. J. \& T. S. Schulenberg (eds). A biological assessment of Parque Nacional Noel Kempff Mercado, Bolivia. Conservation International Washington. RAPWorking Papers 10: 348-355.

Harvey, M. B. \& Gutberlet, R. L., 1998. Lizards of the genus Tropidurus (Iguania: Tropiduridae) from the Serranía de Huanchaca, Bolivia: new species, natural history, and a key to the genus. Herpetologica, 54(4): 493-520. 
Hellmich, W., 1960. Die Sauria des Gran Chaco und seiner Randgebiete. Abh. Bayer. Akad. Wiss. Math.Naturwiss. Kl., 101: 1-131.

Hellmich, W., 1961. Bemerkungen zur geographischen Variabilität von Liolaemus alticolor Barbour (Iguan.). Opuscula Zool., 58: 1-6.

Hellmich, W., 1962. Bemerkungen zur individuellen Varibialität von Liolaemus multiformis (Cope). (Iguanidae.). Opuscula Zool., 67: 1-10.

Hoogmoed, M. S. \& VACA, N., 1990. Herpetofauna. In: Frey, R. (ed.). Report Expedition Perseverancia, 1990: 8-9, 19

HoutTuYN, M., 1782. Het onderschied der salamanderen van de haagdissen in't algemeen, en van de gekkos in't byzonder, aangtond. Verh. Genootsch. Wetenisch Vlissingen, 9: 305-336.

IBISCH, P. \& BöHME, W., 1993. Zur Kenntnis der innerandinen Herpetofauna Boliviens (Provinz Arque, Dep. Cochabamba). Herpetofauna, 15(84): 15-26.

KLuge, A. G., 1964. A revision of the South American gekkonid lizard genus Homonota Gray. Amer. Mus. Nov., 2193: 1-41.

KLuge, A. G., 1969. The evolution and geographical origin of the New World Hemidactylus mabouia-brooki complex (Gekkonidae, Sauria). Misc. Publ. Mus. Zool. Univ. Michigan, 138: 1-78.

Köhler, J., Dirksen, L., Ibisch, P. L., Rauer, G., RudolPh, D. \& BöHME, W., 1995. Zur Herpetofauna des Sehuencas-Bergregenwaldes im CarrascoNationalpark, Bolivien. Herpetofauna, 17(96): 12-25.

KosLOWSKY, J., 1898. Enumeración sistemática y distribución geográfica de los reptiles argentinos. Rev. Mus. La Plata, 8: 161-200.

LAURENT, R. F., 1982. Description de trois nouvelles espèces du genre Liolaemus (Sauria, Iguanidae). Spixiana, 5(2): 139-147.

LAURENT, R. F., 1984. Tres nuevas especies del género Liolaemus (Reptilia, Iguanidae). Acta Zool. Lilloana, 37(2): 273-294.

LAURENT, R. F., 1986. Descripciones de nuevos Iguanidae del genero Liolaemus. Acta Zool. Lilloana, 38(2): 87-105.

LAURENT, R. F., 1995. Sobre una pequeña colección de lagartos del género Liolaemus (Tropiduridae) proveniente del extremo suroeste de Bolivia. Cuad. Herp., 9(1): 1-6.

Lavilla, E. O., GonzÁles, L. \& Fernández, I., 1996. Herpetologia Amboroense. Informe sobre la herpeto fauna del Parque Nacional Amboró y zonas aledañas. Consorcio FAN-TNC. Santa Cruz. 46 pp.

Linnaeus, C., 1758. Systema naturae per regna tria naturae secundum classes, ordines, genera, species sum characteribus differentiis, synonymis, locis. Editia Decima, Reformata. Vol. 1. Laurentii Salvii. Holmiae. 824 pp.
MarX, H., 1960. A new iguanid lizard of the genus Ctenoblepharis. Fieldiana Zool., 39: 407.

Mertens, R., 1929. Herpetologische Mitteilungen. XII. Über einige Amphibien und Reptilien aus SüdBolivien. Zool. Anz., 86: 57-62.

Montero, R., 1996. Amphisbaena bolivica Mertens, 1929, nueva combinación (Squamata: Amphisbaenia). Cuad. Herp., 9(2): 75-84.

Montero, R., Scrocchi, G., Montaño, M. E. \& FERNÁNDEZ S., I. M., 1995. Nuevas citas de saurios, anfisbénidos y ofidios para Bolivia. Cuad. Herp., 9(1): 7-13.

Montero, R., Sáfadez, I. F. \& Álvarez, L. G., 1997. A new species of Amphisbaena from Bolivia. $J$. Herpetol., 31(2): 218-220.

MoreAu DE JonNÉs, A., 1818. Monographie du mabouia des murailles ou Gekko mabouia des Antilles. Bull. Soc. Phil. Paris, 1818: 138-139.

Müller, L., 1923. Über neue oder seltene Mittel- und Südamerikanische Amphibien und Reptilien. Mitt. Zool. Mus. Berlin, 11: 75-93.

MüLlER, L., 1924. Neue oder seltene Reptilien und Batrachier der Zoologischen Sammlung des bayerischen Staates. Zool. Anz., 58: 291-197.

MüLLER, L., 1928. Herpetologische Mitteilungen. Zool. Anz., 77: 61-84.

Ortiz, J. O. \& MArquet, P. L., 1987. Una nueva especie de lagarto altoandino: Liolaemus islugensis (Reptilia-Iguanidae). Gayana, Zool., 51(1/4): 59-63.

O'Shaughnessy, A. W. E., 1879. Descriptions of new species of lizards in the collection of the British Museum. Ann. Mag. Nat. Hist., 4(5): 295-303.

O'Shaughnessy, A. W. E., 1881. An account of the collection of lizards made by Mr. BUCKLEY in Ecuador. Proc. Zool. Soc. London, 1881: 227-245.

PARKer, H. W., 1928a. A new lizard from Argentina. Ann. Mag. Nat. Hist., (10), 2: 383-384.

PARKer, H. W., 1928b. Notes on reptiles and batrachians from Matto Grosso and eastern Bolivia. Ann. Mag. Nat. Hist., (10), 2: 96-97.

Pellegrin, J., 1909. Description de cinq lézards nouveaux des hauts plateaux de Pérou et de la Bolivie. Bull. Mus. Hist. Nat. Paris, 1909: 324-329.

PeraccA, M. G., 1897. Rettili e Anfibi. Viaggio del Dott. Alfredo Borelli nel Chaco boliviano e nella Repubblica Argentina. Boll. Mus. Zool. Anat. Comp. Univ. Torino, 12(274): 1-19.

PÉREz, M. E., 1997. Una evaluación preliminar de los anfibios y reptiles de las Pampas del Heath (Province Iturralde, Departamento de La Paz). Ecol. Bolivia, 30: $43-54$.

Pérez-Mellado, V. \& De la Riva, I., 1993. Sexual dimorphism and ecology: the case of a tropical 
lizard, Tropidurus melanopleurus (Sauria, Tropiduridae). Copeia, 1993(4): 969-976.

Peters, J. A. \& Donoso-BArros, R., 1970. Catalogue of neotropical Squamata. Part II. Lizards and amphisbaenians. U. S. Natl. Mus. Bull., 297: v-viii, 1293.

Peters, W., 1862. Über Cercosaura und die mit dieser verwandten Eidechsen aus Südamerika. Monatsber. Königl. Akad. Wiss. Berlin, 1862: 165-225.

Peters, W., 1887. Über die von SPIX in Brasilien gesammelten Eidechsen des Königlichen NaturalienKabinets zu München. Monatsber. Königl. Akad. Wiss. Berlin, 1887: 407-423.

Presch, W., 1973. A review of the tejus lizard genus Tupinambis (Sauria: Teiidae) from South America. Copeia, 1973(4): 740-746.

Procter, J. B., 1921. On a small collection of reptiles and batrachians made by Mr. GoOdFELLOw in eastern Bolivia (1918-1919). Ann. Mag. Nat. Hist., 9(7): 189-193.

ReInHARDT, J. \& LÜTKEN, C., 1862. Bidrag til Kundskab om Brasiliens Padder og Krybdyr. Vidensk. Medd. Dansk Naturhist. Foren., 3(1861): 141-242.

RENDAHL, H., 1937. Einige Reptilien aus Ekuador und Bolivien. Ark. Zool., 29(13): 1-19.

Rhodes, C., 1963. On Amphisbaena fuliginosa Linné from Bolivia. Herpetologica, 19(3): 175-178.

RodriguEs, M. T., 1987. Sistemática, ecologia e zoogeografia dos Tropidurus do grupo torquatus ao sul do rio Amazonas (Sauria, Iguanidae). Arq. Zool. São Paulo, 31(3): 105-230.

RuibAL, R., 1952. Revisionary studies of some South American Teiidae. Bull. Mus. Comp. Zool., 106(1): 477-529.

Schmidt, K. P. \& Inger, R. F., 1951. Amphibians and reptiles of the Hopkins-Branner expedition to Brazil. Fieldiana Zool., 31(42): 439-465.

Scrocchi, G. \& GonzÁles, L., 1996. Kentropyx calca rata (NCN). Behavior. Herp. Rev., 27(4): 201.

SCrocchi, G. \& GonzÁles, L., 1996. Informe sobre la herpetofauna del Parque Nacional Noel Kempff Mercado, Santa Cruz - Bolivia. Consorcio FANTNC. Santa Cruz. 32 pp.

SinITSIN, D. T., 1930. Description of a new species of Neusticurus from South America (Lizards, Teiidae). Amer. Mus. Nov., 408: 1-7.

Smith, H. M., Martin, R. L. \& Swain, T. A., 1977. A new genus and two new species of South American geckos (Reptilia: Lacertilia). Pap. Avul. Zool. São Paulo, 30(14): 195-213.

SpIX, J. B., 1825. Animalia nova sive species novae lacertarum quas in itinere per Brasiliam anni 18171820 jussu auspiciis Maximiliani Josephi Bavaria Regis. Tomus I. Lacertae. Vol. I. Leipzig. iv + 26 pp.
STRAuCH, A., 1883. Bemerkungen über Eidechsenfamilien der Amphisbaeniden. Bull. Acad. Imp. Sci. St. Petersburg. 28: 45-131.

Tedesco, M. E. \& CeI, J. M., 1997. Osteological research on the genus Kentropyx from Argentina and revalidation of the specific status for Kentropyx lagartija (Gallardo, 1962) (Squamata: Teiidae). Boll. Mus. reg. Sci. Nat. Torino, 15(1): 231-240.

TORRES, S. E., 1996. Urostrophus gallardoi: Geographic Distribution. Herp. Rev., 27(4): 212.

Uzzell, T. M., 1966. Teeid lizards of the genus Neusticurus (Reptilia, Sauria). Bull. Amer. Mus. Nat. Hist., 132(5): 279-327.

Uzzell, T. M., 1969. A new genus and species of teiid lizard from Bolivia. Postilla, 129: 1-15.

Uzzell, T. M., 1970. Teiid lizards of the genus Proctoporus from Bolivia and Peru. Postilla, 142: 139.

Uzzell, T. M., 1973. A revision of the genus Prionodactylus with a new genus for P. leucostictus and notes on the genus Euspondylus (Sauria, Teiidae). Postilla, 159: 1-67.

VAnzolini, P. E., 1961. Bachia: Especies brasileiras e conceito genérico (Sauria: Teiidae). Pap. Dep. Zool. Sec. Agric. São Paulo, 14: 193-209.

VAnzolini, P. E., 1986. Addenda and corrigenda to the catalogue of neotropical Squamata. Smithson. Herpetol. Inform. Serv., 70: 1-25.

VAnzolini, P. E., 1992. Cercolophia, a new genus for the species of Amphisbaena with a terminal vertical keel on the tail (Reptilia, Amphisbaenia). Pap. Avul. Zool., São Paulo, 37(27): 401-412.

VAnZOLINI, P. E., 1995. Neusticurus ocellatus, 1930: A valid species of teeid lizard from Bolivia. Amer. Mus. Nov., 3123: 1-7.

Veloso, A., Ortíz, J. C., Navarro., J., Núñez, H., Espejo, P. \& LABra, M. A., 1995. Reptiles. In: Simmonetti et al. (ed): Diversidad biológica en Chile. Comité Nacional de Diversidad Biológica, CONICYT.: 326-335.

WAGLer, J., 1824. Serpentum brasiliensium species novae. In: Spix, J. de. Animalia nova sive species novae testidinum et ranarum. Monachi: viii +75 .

Wagler, J., 1830. Natürliches System der Amphibien, mit vorhandener Classification der Säugethiere und Vögel. München. 354 pp.

Werner, F., 1899. Beschreibung neuer Reptilien und Batrachier. Zool. Anz., 22: 479-483.

Werner, F., 1910. Über neue oder seltene Reptilien des Naturhistorischen Museums in Hamburg. II. Eidechsen. Mitt. Naturhist. Mus. Hamburg, 27: 1-46.

Werner, F., 1913. Neue oder seltene Reptilien und Frösche des Naturhistorischen Museums in Hamburg. Mitt. Naturhist. Mus. Hamburg, 30(1912) 2: $1-51$. 
WIED-Neuwied, M., 1820. Reise nach Brasilien in den Jahren 1815 bis 1817. Vol. I. Frankfurt am Main. xxxiv $+380 \mathrm{pp}$.

Wiegmann, A. F., 1834. Herpetologica mexicana. Seu descriptio amphibiorum Novae Hispaniae, quae iti neribus comitis de Sack, Fernando Deppe, et Chr. Schiede. Vol. 1. Saurorum species. Berlin. vii +54 pp.

Appendix 1.- Gazetteer. *Localities not found.

Apéndice 1.- Lista de localidades de Bolivia. *Localidades no encontradas.

\section{Department of Beni}

$\underline{\text { LOCALITY }}$

Arroyo Grande

Beni Biosphere Reserve

Cachuela Esperanza

Camiaco

Chacabo Indian Village

Costa Márquez

El Remanso

Estación Biológica Beni

Estancia Edén, 25 km SWSan Joaquín

Estancia El Porvenir

Estancia La Cabaña

Estancia Venecia

Estancia Yutiole, 20 km S San Joaquín

Exaltación

Guayaramerín

Ivón

Lago Victoria

La Rangeira, Río Iténez

Loreto

Magdalena

Pacusal

Pampa de Meio

Puerto Almacén

Puerto Caballo

Puerto Cap. Vázquez, Río Iténez

Puerto Moré

Puerto Siles

Puerto Versalles

Rancho Espíritu

Rancho San Marcos

Reyes

Riberalta

Río Beni

Río Blanco, 4-5 km from confluence w. Río Iténez

Río Curiraba, Beni Biosphere Reserve

Río Curichá

Río Isiboro

Río Iténez

Río Maniquí

Río Mamoré, between Loreto and the mouth of Río Sara

Río Mamoré

Río Mamoré, 10 km E of San Antonio

Río Quiquibey

Río Sara

\begin{tabular}{|c|c|}
\hline & Río Yacuma \\
\hline$\underline{\text { COORDINATES }}(\mathrm{S} / \mathrm{W})$ & Río Yata \\
\hline $10^{\circ} 49^{\prime} / 65^{\circ} 25^{\prime}$ & Rurrenabaque \\
\hline $14^{\circ} 51^{\prime} / 62^{\circ} 21^{\prime}$ & San Antonio \\
\hline $\begin{array}{l}14.51 / 02.21 \\
10^{\circ} 32^{\prime} / 65^{\circ} 38^{\prime},\end{array}$ & San Borja \\
\hline $15^{\circ} 19^{\prime} / 64^{\circ} 44^{\prime}$ & San Javier \\
\hline $\begin{array}{l}15^{\circ} 19^{\prime} / 64^{\circ} 44^{\prime} \\
12^{\circ} 20^{\prime} / 66^{\circ} 00^{\prime}\end{array}$ & San Joaquín \\
\hline $\begin{array}{l}12^{\circ} 20^{\prime} / 66^{\circ} 00^{\prime} \\
12^{\circ} 29^{\prime} / 64^{\circ} 17^{\prime}\end{array}$ & San Pedro \\
\hline $12^{\circ} 29^{\prime} / 64^{\circ} 17^{\prime}$, & Santa Fé, Río Iténez \\
\hline $13^{\circ} 31^{\prime} / 61^{\circ} 53^{\prime}$ & Santa Rosa, Río Mamoré \\
\hline $14^{\circ} 51^{\prime} / 62^{\circ} 21^{\prime}$ & Trinidad \\
\hline $13^{\circ} 09^{\prime} / 64^{\circ} 50^{\prime}$ & Tumi Chucua \\
\hline $14^{\circ} 38^{\prime} / 66^{\circ} 18^{\prime}$ & Villa Bella \\
\hline $15^{\circ} 03^{\prime} / 66^{\circ} 58^{\prime}$ & Villa DCHa \\
\hline $14^{\circ} 40^{\prime} / 65^{\circ} 24^{\prime}$ & Department of Chuquisaca \\
\hline $13^{\circ} 13^{\prime} / 64^{\circ} 40^{\prime}$ & \\
\hline $13^{\circ} 16^{\prime} / 65^{\circ} 15^{\prime}$ & $\frac{\text { LOCALITY }}{\text { Corandouti }}$ \\
\hline $10^{\circ} 49^{\prime} / 66^{\circ} 51^{\prime}$ & Carandayti \\
\hline $11^{\circ} 08^{\prime} / 66^{\circ} 07^{\prime}$ & Charobamba \\
\hline $10^{\circ} 59^{\prime} / 66^{\circ} 10^{\prime}$ & Chichas \\
\hline $13^{\circ} 19^{\prime} / 62^{\circ} 00^{\prime}$ & El Palmar \\
\hline $15^{\circ} 13^{\prime} / 64^{\circ} 40^{\prime}$ & E1 Salvador \\
\hline $13^{\circ} 15^{\prime} / 64^{\circ} 03^{\prime}$ & Pampa Ruiz \\
\hline $13^{\circ} 10^{\prime} / 62^{\circ} 07^{\prime}$ & Paso de Macho Cruz \\
\hline $12^{\circ} 30^{\prime} / 64^{\circ} 15^{\prime}$ & Pescado (= Villa Serrano) \\
\hline $14^{\circ} 52^{\prime} / 64^{\circ} 58^{\prime}$ & Río Grande, entre Valle Grande y Pescado \\
\hline $\begin{array}{c}13^{\circ} 42^{\prime} / 65^{\circ} 20^{\prime} \\
*\end{array}$ & Sucre \\
\hline $12^{\circ} 00^{\prime} / 65^{\circ} 06^{\prime}$ & Department of Cochabamba \\
\hline $12^{\circ} 47^{\prime} / 65^{\circ} 02^{\prime}$ & LOCALITY \\
\hline $12^{\circ} 41^{\prime} / 63^{\circ} 22^{\prime}$ & $\underline{\text { LUCALIIY }}$ \\
\hline $14^{\circ} 13^{\prime} / 66^{\circ} 40^{\prime}$ & Arani \\
\hline $15^{\circ} 28^{\prime} / 65^{\circ} 05^{\prime}$ & Boca Chapare \\
\hline $14^{\circ} 19^{\prime} / 67^{\circ} 23^{\prime}$ & Challa \\
\hline $11^{\circ} 00^{\prime} / 66^{\circ} 04^{\prime}$ & Charuplaya \\
\hline $10^{\circ} 23^{\prime} / 65^{\circ} 24^{\prime}$ & Chipiriri \\
\hline $12^{\circ} 31^{\prime} / 64^{\circ} 17^{\prime}$ & Cliza \\
\hline $14^{\circ} 50^{\prime} / 66^{\circ} 23^{\prime}$ & Cochabamba, Cd.; $2600 \mathrm{~m}$ \\
\hline $12^{\circ} 32^{\prime} / 63^{\circ} 55^{\prime}$ & El Palmar \\
\hline $15^{\circ} 28^{\prime} / 65^{\circ} 05^{\prime}$ & Incachaca \\
\hline $\begin{array}{l}15 \cdot 28 / 0505 \\
11^{\circ} 28^{\prime} / 65^{\circ} 05^{\prime}\end{array}$ & La Siberia \\
\hline $\begin{array}{l}11.28,0505 \\
14^{\circ} 49^{\prime} / 66^{\circ} 51^{\prime},\end{array}$ & Locotal \\
\hline $1449 / 0051$ & Mizque \\
\hline $10^{\circ} 23^{\prime} / 65^{\circ} 23^{\prime}$ & Monte Punco \\
\hline $13^{\circ} 35^{\prime} / 65^{\circ} 19^{\prime}$ & Monterredondo \\
\hline $12^{\circ} 26^{\prime} / 65^{\circ} 10^{\prime}$ & Paracti \\
\hline 122070510 & Parotani \\
\hline $\begin{array}{l}143176 / 35 \\
15^{\circ} 51, / 64^{\circ} 39^{\prime}\end{array}$ & Peña Colorada \\
\hline & Pocona \\
\hline
\end{tabular}

$13^{\circ} 38^{\prime} / 62^{\circ} 53^{\prime}$

$10^{\circ} 59^{\prime} / 65^{\circ} 35$

$14^{\circ} 28^{\prime} / 67^{\circ} 34^{\prime}$

$13^{\circ} 56^{\prime} / 65^{\circ} 08^{\prime}$

$14^{\circ} 49^{\prime} / 66^{\circ} 51^{\prime}$

$14^{\circ} 36^{\prime} / 64^{\circ} 53^{\prime}$ '

$13^{\circ} 02^{\prime} / 64^{\circ} 40^{\prime}$

$14^{\circ} 00^{\prime} / 65^{\circ} 04^{\prime}$

$11^{\circ} 33^{\prime} / 65^{\circ} 16^{\prime}$

$11^{\circ} 08^{\prime} / 66^{\circ} 10^{\prime}$

$11^{\circ} 08^{\prime} / 66^{\circ} 10^{\prime}$

$10^{\circ} 23^{\prime} / 65^{\circ} 24^{\prime}$

CoOrdinates $(\mathrm{S} / \mathrm{W})$

$20^{\circ} 45^{\prime} / 63^{\circ} 04^{\prime}$

*

$20^{\circ} 51^{\prime} / 64^{\circ} 19^{\prime}$

$20^{\circ} 45^{\prime} / 63^{\circ} 04^{\prime}$

$20^{\circ} 45^{\prime} / 65^{\circ} 15^{\prime}$

$*$

$19^{\circ} 06^{\prime} / 64^{\circ} 22^{\prime}$

$15^{\circ} 51^{\prime} / 64^{\circ} 39^{\prime}$

$19^{\circ} 02^{\prime} / 65^{\circ} 17^{\prime}$

COORDINATES $(\mathrm{S} / \mathrm{W})$

$17^{\circ} 34^{\prime} / 65^{\circ} 47^{\prime}$

$15^{\circ} 58^{\prime} / 64^{\circ} 41^{\prime}$

$17^{\circ} 37^{\prime} / 66^{\circ} 40^{\prime}$

$16^{\circ} 36^{\prime} / 66^{\circ} 37^{\prime}$

$16^{\circ} 13^{\prime} / 65^{\circ} 15^{\prime}$

$17^{\circ} 36^{\prime} / 65^{\circ} 56^{\prime}$

$17^{\circ} 24^{\prime} / 65^{\circ} 48^{\prime}$

$17^{\circ} 06^{\prime} / 65^{\circ} 32^{\prime}$

$17^{\circ} 14^{\prime} / 65^{\circ} 49^{\prime}$

$17^{\circ} 50^{\prime} / 64^{\circ} 45^{\prime}$,

$17^{\circ} 11^{\prime} / 65^{\circ} 48^{\prime}$

$17^{\circ} 57^{\prime} / 65^{\circ} 20^{\prime}$

$17^{\circ} 35^{\prime} / 65^{\circ} 17^{\prime}$

$17^{\circ} 34^{\prime} / 66^{\circ} 61^{\prime}$

$17^{\circ} 12^{\prime} / 65^{\circ} 49^{\prime}$

$17^{\circ} 34^{\prime} / 66^{\circ} 21^{\prime}$,

$18^{\circ} 10^{\prime} / 64^{\circ} 52^{\prime}$

$17^{\circ} 39^{\prime} / 65^{\circ} 24^{\prime}$ 


Puerto San Mateo
Quiroga
Río Chua Khocha
Sehuencas
Tablasmontes
Tiraque
Totora
Tucachaca
Valle de Sajta
Villa Granado
Villa Tunari
Viña Perdida Farm
Department of La Paz
LocALITY
Achacachi
Achocalla
Alcoche
Alto Següencoma
Barraca
Calabatea
Caracoles
Caranavi
Caupolicán
Chacaltaya
Chaco
Cheripa
Chilalaya (= Chililaya)
Chulumani
Collana
Confluence of the Río Kaka and the Río Beni
Cordillera de Apolobamba
Cordillera de Irupana
Coroico
La Cumbre
El Tambillo, Río Pallina
Esperanza, Province Pacajes
Hacienda Collana, entre Ayo-Ayo y Calamarca
Hacienda Corpaputu, 10 km E Achacachi
Huachi
Huacullani
Huajchilla
Huaraco
Huatajata
Ixiamas
Jacha Toloko
Jupapina
Kallutaca
Lago Titicaca
La Paz, Cd.
La Paz, entre La Paz y Oruro
Lipari
Llojeta
Mapiri
Mallasa
Misiones Mosetenes (= Covendo ?)
Palca
Pampas del Heath
Patacamaya
Patamanta
Pelechuco
Potone
Potamanta
Pucarani
Puerto Linares
Querqueta
Reserva Ulla Ulla
Alto Achachicala, Río Caluyo

\begin{tabular}{|c|c|}
\hline & \\
\hline $17^{\circ} 42^{\prime} / 64^{\circ} 47^{\prime}$ & Río Chimate \\
\hline $18^{\circ} 25^{\prime} / 65^{\circ} 13^{\prime}$ & Río Irpavi, near Karpani \\
\hline $17^{\circ} 47^{\prime} / 64^{\circ} 42^{\prime}$ & Río Kaka \\
\hline $17^{\circ} 30^{\prime} / 65^{\circ} 16^{\prime}$ & Río Machariapo \\
\hline $17^{\circ} 07^{\prime} / 66^{\circ} 01^{\prime}$ & Río Madidi \\
\hline $17^{\circ} 25^{\prime} / 65^{\circ} 43^{\prime}$ & Río Mapiri \\
\hline $17^{\circ} 44^{\prime} / 65^{\circ} 12^{\prime}$ & Río Ovejuyo \\
\hline$*$ & Río Suapi \\
\hline $17^{\circ} 00^{\prime} / 64^{\circ} 50^{\prime}$ & Río Zongo (= Songo) \\
\hline $18^{\circ} 12^{\prime} / 65^{\circ} 02^{\prime}$ & San Antonio \\
\hline $16^{\circ} 57^{\prime} / 65^{\circ} 24^{\prime}$ & San Buenaventura \\
\hline & San Fermín \\
\hline & San José \\
\hline & Sorata \\
\hline COORDINATES $(\mathrm{S} / \mathrm{W})$ & Tamampoya (= Tamanpaya) \\
\hline $16^{\circ} 02^{\prime} / 68^{\circ} 41^{\prime}$ & Taraco, Península \\
\hline $\begin{array}{l}10^{\circ} 02768^{\circ} 41 \\
16^{\circ} 35^{\prime} / 68^{\circ} 10^{\prime}\end{array}$ & Tihuanacu (= Tiahuanaco, Tiwanaku) \\
\hline $15^{\circ} 44^{\prime} / 67^{\circ} 40^{\prime}$ & Tipuani \\
\hline $\begin{array}{c}40 / 40 \\
*\end{array}$ & Tumupassa \\
\hline $12^{\circ} 32^{\prime} / 66^{\circ} 52^{\prime}$ & Ulla Ulla \\
\hline $14^{\circ} 58^{\prime} / 68^{\circ} 30^{\prime}$ & Unduavi \\
\hline $16^{\circ} 55^{\prime} / 67^{\circ} 19^{\prime}$ & Upper Río Madidi \\
\hline $15^{\circ} 46^{\prime} / 67^{\circ} 36^{\prime}$ & Villa Holguín \\
\hline$*$ & Yanacachi \\
\hline $16^{\circ} 20^{\prime} / 68^{\circ} 08^{\prime}$ & Yolosa \\
\hline $16^{\circ} 21^{\prime} / 67^{\circ} 49^{\prime}$ & Zongo Valley \\
\hline $16^{\circ} 13^{*} / 68^{\circ} 27$ & Department of Oruro \\
\hline $16^{\circ} 24^{\prime} / 67^{\circ} 43$ & $\underline{\text { LOCALITY }}$ \\
\hline $16^{\circ} 38^{\prime} / 68^{\circ} 58^{\prime}$ & Caracollo \\
\hline $15^{\circ} 29^{\prime} / 67^{\circ} 50^{\prime}$ & Challapata \\
\hline $14^{\circ} 46^{\prime} / 68^{\circ} 27^{\prime}$ & Crequi \\
\hline $16^{\circ} 28^{\prime} / 67^{\circ} 28^{\prime}$ & Eucaliptus \\
\hline $16^{\circ} 10^{\prime} / 67^{\circ} 44^{\prime}$ & Hacienda Huancaroma \\
\hline $16^{\circ} 23^{\prime} / 67^{\circ} 23^{\prime}$ & Lago Poopo \\
\hline $16^{\circ} 33^{\prime} / 68^{\circ} 31^{\prime}$ & Oruro, Cd. \\
\hline $14^{\circ} 42^{\prime} / 66^{\circ} 45^{\prime}$ & Playa Verde \\
\hline $17^{\circ} 04^{\prime} / 68^{\circ} 05^{\prime}$ & Senechal \\
\hline $16^{\circ} 02^{\prime} / 68^{\circ} 38^{\prime}$ & \\
\hline $15^{\circ} 40^{\prime} / 67^{\circ} 14^{\prime}$ & Department of Pando \\
\hline $14^{\circ} 46^{\prime} / 66^{\circ} 52^{\prime}$ & LOCALITY \\
\hline $16^{\circ} 38^{\prime} / 68^{\circ} 04^{\prime}$ & \\
\hline $17^{\circ} 22^{\prime} / 67^{\circ} 37^{\prime}$ & Abuná \\
\hline $16^{\circ} 11^{\prime} / 68^{\circ} 44^{\prime}$ & Cobija \\
\hline $13^{\circ} 45^{\prime} / 68^{\circ} 09^{\prime}$ & Ingavi \\
\hline $16^{\circ} 21^{\prime} / 68^{\circ} 02^{\prime}$ & Mucden (= Mukden) \\
\hline $16^{\circ} 38^{\prime} / 68^{\circ} 03^{\prime}$ & Puesto Abuná \\
\hline$*$ & Río Nareuda \\
\hline $15^{\circ} 48^{\prime} / 69^{\circ} 24^{\prime}$ & San Juan del Nuevo Mundo \\
\hline $16^{\circ} 30^{\prime} / 68^{\circ} 09^{\prime}$ & \\
\hline $16^{\circ} 49^{\prime} / 68^{\circ} 11^{\prime}$ & Department of Potosí \\
\hline $16^{\circ} 37^{\prime} / 68^{\circ} 02^{\prime}$ & $\underline{\text { LOCALITY }}$ \\
\hline $16^{\circ} 32^{\prime} / 68^{\circ} 08^{\prime}$ & Campamento Khastor \\
\hline $15^{\circ} 15^{\prime} / 68^{\circ} 10^{\prime}$ & Chichas \\
\hline $16^{\circ} 34^{\prime} / 68^{\circ} 05^{\prime}$ & Chocaya \\
\hline $15^{\circ} 47^{\prime} / 67^{\circ} 05^{\prime}$ & Cordillera Chichas \\
\hline $16^{\circ} 34^{\prime} / 67^{\circ} 59^{\prime}$ & Julaca \\
\hline $13^{\circ} 01^{\prime} / 68^{\circ} 50^{\prime}$ & Laguna Colorada \\
\hline $17^{\circ} 14^{\prime} / 67^{\circ} 55^{\prime}$ & Paso de Macho Cruz \\
\hline $16^{\circ} 22^{\prime} / 68^{\circ} 22^{\prime}$ & Portugalete \\
\hline $14^{\circ} 48^{\prime} / 69^{\circ} 04^{\prime}$ & Potosí, Cd. \\
\hline * & Salar de Chalvirí \\
\hline $17^{\circ} 10^{\prime} / 68^{\circ} 10^{\prime}$ & Uyuni \\
\hline $16^{\circ} 24^{\prime} / 68 ! 29^{\prime}$ & Villazón \\
\hline $15^{\circ} 30^{\prime} / 67^{\circ} 31^{\prime}$ & \\
\hline $16^{\circ} 36^{\prime} / 68^{\circ} 34^{\prime}$ & Department of Santa Cruz \\
\hline $15^{\circ} 03^{\prime} / 69^{\circ} 16^{\prime}$ & LOCALITY \\
\hline $16^{\circ} 20^{\prime} / 68^{\circ} 06^{\prime}$ & \\
\hline
\end{tabular}

$15^{\circ} 25^{\prime} / 68^{\circ} 00^{\prime}$

$15^{\circ} 10^{\prime} / 67^{\circ} 44^{\prime}$ $14^{\circ} 36^{\prime} / 68^{\circ} 35^{\prime}$ $12^{\circ} 32^{\prime} / 66^{\circ} 52^{\prime}$ $15^{\circ} 15^{\prime} / 68^{\circ} 16^{\prime}$, $16^{\circ} 32^{\prime} / 68^{\circ} 02^{\prime}$ $15^{\circ} 30^{\prime} / 67^{\circ} 31^{\prime}$ $16^{\circ} 07^{\prime} / 68^{\circ} 02^{\prime}$ $14^{\circ} 34^{\prime} / 68^{\circ} 22^{\prime}$ $14^{\circ} 27^{\prime} / 67^{\circ} 33^{\prime}$ $13^{\circ} 58^{\prime} / 68^{\circ} 58^{\prime}$ $16^{\circ} 21^{\prime} / 68^{\circ} 40^{\prime}$ $15^{\circ} 47^{\prime} / 68^{\circ} 40^{\prime}$ $16^{\circ} 16^{\prime} / 67^{\circ} 13^{\prime}$ $15^{\circ} 47^{\prime} / 68^{\circ} 40^{\prime}$ $16^{\circ} 33^{\prime} / 68^{\circ} 42^{\prime}$ $15^{\circ} 33^{\prime} / 68^{\circ} 00^{\prime}$ $14^{\circ} 09^{\prime} / 67^{\circ} 55^{\prime}$ $15^{\circ} 03^{\prime} / 69^{\circ} 16^{\prime}$ $16^{\circ} 19^{\prime} / 67^{\circ} 55^{\prime}$ $13^{\circ} 35^{\prime} / 68^{\circ} 46^{\prime}$ $16^{\circ} 23^{\prime} / 67^{\circ} 49^{\prime}$ $16^{\circ} 14^{\prime} / 67^{\circ} 43^{\prime}$ $16^{\circ} 06^{\prime} / 68^{\circ} 03^{\prime}$

Coordinates (S/W)

$17^{\circ} 38^{\prime} / 67^{\circ} 13^{\prime}$ $15^{\circ} 53^{\prime} / 68^{\circ} 07^{\prime}$ $18^{\circ} 52^{\prime} / 67^{\circ} 18^{\prime}$ $17^{\circ} 35^{\prime} / 67^{\circ} 31^{\prime}$ $17^{\circ} 40^{\prime} / 67^{\circ} 29^{\prime}$ $18^{\circ} 45^{\prime} / 67^{\circ} 07^{\prime}$ $17^{\circ} 58^{\prime} / 67^{\circ} 06^{\prime}$ *

COORDINATES (S/W) $09^{\circ} 42^{\prime} / 65^{\circ} 23^{\prime}$ $11^{\circ} 02^{\prime} / 68^{\circ} 44^{\prime}$ $10^{\circ} 57^{\prime} / 66^{\circ} 50^{\prime}$ $11^{\circ} 11^{\prime} / 69^{\circ} 02^{\prime}$ $09^{\circ} 47^{\prime} / 65^{\circ} 32^{\prime}$ $11^{\circ} 08^{\prime} / 68^{\circ} 46^{\prime}$ $10^{\circ} 46^{\prime} / 64^{\circ} 44^{\prime}$

COORDinates (S/W) $22^{\circ} 02^{\prime} / 66^{\circ} 08^{\prime}$ $20^{\circ} 53^{\prime} / 66^{\circ} 17^{\prime}$ $20^{\circ} 30^{\prime} / 66^{\circ} 30^{\prime}$ $20^{\circ} 54^{\prime} / 67^{\circ} 34^{\prime}$ $22^{\circ} 12^{\prime} / 67^{\circ} 46^{\prime}$ $*$

$20^{\circ} 52^{\prime} / 66^{\circ} 07^{\prime}$ $19^{\circ} 35^{\prime} / 65^{\circ} 45^{\prime}$ $22^{\circ} 32^{\prime} / 67^{\circ} 34^{\prime}$ $20^{\circ} 28^{\prime} / 66^{\circ} 50^{\prime}$ $22^{\circ} 06^{\prime} / 65^{\circ} 37^{\prime}$

COORDinATES (S/W) $18^{\circ} 50^{\prime} / 63^{\circ} 28^{\prime}$ 


Abra de la Cruz
Aguaraigua
Airport Viru Viru
Asunción
Ayacucho, 16 km SWof Santa Cruz
Ayacucho de Yapacaní
Boyuibi
Buena Vista
Camiri
Campamento Ichilo
Campamento Las Torres
Campamento Macuñucú
Campamento, Río San Martin
Caranda
Cerro Colorado
Cerro Hosane
Charagua
10 km S of Colonia San Martín
Concepción
Comarapa
Comunidad Guirapembi
Cotoca
Cupesí
Curuyuqui
El Carmen
El Fuerte (Samaipata)
El Portón
El Refugio
Espejillos
Esperanza
Finca de Milanos
Flor de Oro
Florida
Guadalupe
Huasacañada
Inselberg
La Brecha
Lago Caimán
La Guitarra
Lake Gahibia-Mirim
La Madre
Las Juntas
Lomas de Arena
Los Fierros
Los Troncos
Mataral
Meseta de Caparuch
Naranjos
Nueva Moka
Okinawa 1
Pampa Grande
Pampas Unión
Parque Nacional Amboró

$17^{\circ} 50^{\prime} / 64^{\circ} 20$

$19^{\circ} 24^{\prime} / 62^{\circ} 48^{\prime}$

$17^{\circ} 40^{\prime} / 63^{\circ} 08^{\prime}$

$17^{\circ} 50^{\prime} / 60^{\circ} 10^{\prime}$

$17^{\circ} 51^{\prime} / 63^{\circ} 18^{\prime}$

$17^{\circ} 22^{\prime} / 63^{\circ} 50$ '

$20^{\circ} 17^{\prime} / 63^{\circ} 17^{\prime}$

$17^{\circ} 27^{\prime} / 63^{\circ} 40^{\prime}$

$20^{\circ} 02^{\prime} / 63^{\circ} 30^{\prime}$

$17^{\circ} 24^{\prime} / 64^{\circ} 14^{\prime}$

$13^{\circ} 39^{\prime} / 60^{\circ} 48^{\prime}$

$17^{\circ} 43^{\prime} / 63^{\circ} 35^{\prime}$

$15^{\circ} 03^{\prime} / 61^{\circ} 56^{\prime}$

$17^{\circ} 32^{\prime} / 63^{\circ} 31^{\prime}$

$19^{\circ} 28^{\prime} / 62^{\circ} 21^{\prime}$

$17^{\circ} 25^{\prime} / 64^{\circ} 00^{\prime}$

$19^{\circ} 48^{\prime} / 63^{\circ} 13^{\prime}$

$15^{\circ} 29^{\prime} / 61^{\circ} 38^{\prime}$

$16^{\circ} 15^{\prime} / 62^{\circ} 24^{\prime}$

$17^{\circ} 55^{\prime} / 64^{\circ} 32^{\prime}$

$19^{\circ} 26^{\prime} / 62^{\circ} 21^{\prime}$

$17^{\circ} 49^{\prime} / 63^{\circ} 03^{\prime}$

$18^{\circ} 28^{\prime} / 62^{\circ} 04^{\prime}$

$18^{\circ} 46^{\prime} / 62^{\circ} 14^{\prime}$

$18^{\circ} 48^{\prime} / 58^{\circ} 33^{\prime}$

$18^{\circ} 10^{\prime} / 63^{\circ} 50^{\prime}$

$18^{\circ} 06^{\prime} / 60^{\circ} 03^{\prime}$

$14^{\circ} 45^{\prime} / 61^{\circ} 00^{\prime}$

$17^{\circ} 50^{\prime} / 63^{\circ} 25^{\prime}$

$16^{\circ} 28^{\prime} / 61^{\circ} 16^{\prime}$

$17^{\circ} 50^{\prime} / 60^{\circ} 45^{\prime}$

$13^{\circ} 33^{\prime} / 61^{\circ} 00^{\prime}$

$14^{\circ} 37^{\prime} / 61^{\circ} 12$ '

$18^{\circ} 33^{\prime} / 64^{\circ} 05^{\prime}$

$18^{\circ} 31^{\prime} / 64^{\circ} 05^{\prime}$

$14^{\circ} 35^{\prime} / 61^{\circ} 31^{\prime}$

$19^{\circ} 30^{\prime} / 62^{\circ} 34^{\prime}$

$13^{\circ} 35^{\prime} / 60^{\circ} 54^{\prime}$

$18^{\circ} 06^{\prime} / 63^{\circ} 32^{\prime}$

$17^{\circ} 45^{\prime} / 57^{\circ} 43^{\prime}$

$18^{\circ} 52^{\prime} / 62^{\circ} 21^{\prime}$

$18^{\circ} 38^{\prime} / 63^{\circ} 08^{\prime}$

$17^{\circ} 48^{\prime} / 63^{\circ} 10^{\prime}$

$14^{\circ} 33^{\prime} / 60^{\circ} 55^{\prime}$

$16^{\circ} 40^{\prime} / 61^{\circ} 57^{\prime}$

$18^{\circ} 05^{\prime} / 64^{\circ} 11^{\prime}$

$13^{\circ} 54^{\prime} / 60^{\circ} 49^{\prime}$

$18^{\circ} 38^{\prime} / 59^{\circ} 09^{\prime}$

$17^{\circ} 19^{\prime} / 63^{\circ} 33^{\prime}$

$17^{\circ} 13^{\prime} / 62^{\circ} 54^{\prime}$

$18^{\circ} 05^{\prime} / 64^{\circ} 06^{\prime}$

$17^{\circ} 48^{\prime} / 63^{\circ} 21^{\prime}$

$17^{\circ} 40^{\prime} / 63^{\circ} 15^{\prime}$
Perforación

Perseverancia

Piso Firme

Puerto Almacén

Puerto Suárez

Remates, $\mathrm{N}$ of San Juan del Potrero

Reserva Nacional de Flora y Fauna de Tariquía

Río Cheyo

Río Saguayo

Río Seco (45 km W); 700 m

Río Surutú

Roboré

Samaipata

San Antonio de Parapet

San Ignacio de Velasco

San Javier

San José de Chiquitos

San Juan del Potrero

San Lorenzo

San Pablo

San Ramón

Santa Cruz de la Sierra

Santa Elena

Santa Rosa de la Roca

Santiago

Serranía de Huanchaca

Sounaiporta (probably Samaipata)

Tierras Nuevas

Valle Grande (= Vallegrande)

Airport Viru Viru

Yapacaní

Yapiroa

Warnes

Department of Tarija

LOCALITY

Aguairenda

Border of Tarija River, entry to Salinas

Caiza (= Villa Ingavi)

Capirenda

Catamarca

Entre Ríos

Fortin Margariños

Río Pilcomayo

Río Seco

Salinas

San Francisco (= Villa Montes)

Sunchal

Taringuiti

Tarairi

Tarija, Cd.

Villa Montes $19^{\circ} 55^{\prime} / 62^{\circ} 33^{\prime}$

$14^{\circ} 18^{\prime} / 63^{\circ} 10^{\prime}$

$13^{\circ} 38^{\prime} / 61^{\circ} 45^{\prime}$

$15^{\circ} 46^{\prime} / 62^{\circ} 15^{\prime}$

$18^{\circ} 58^{\prime} / 57^{\circ} 48$

$17^{\circ} 53^{\prime} / 64^{\circ} 20^{\prime}$

$21^{\circ} 56^{\prime} / 64^{\circ} 19^{\prime}$

$17^{\circ} 40^{\prime} / 63^{\circ} 39^{\prime}$

$17^{\circ} 35^{\prime} / 63^{\circ} 48^{\prime}$

$18^{\circ} 35^{\prime} / 63^{\circ} 33^{\prime}$

$17^{\circ} 24^{\prime} / 63^{\circ} 51^{\prime}$

$18^{\circ} 20^{\prime} / 59^{\circ} 45^{\prime}$

$18^{\circ} 10^{\prime} / 64^{\circ} 53^{\prime}$

$20^{\circ} 01^{\prime} / 63^{\circ} 13^{\prime}$

$16^{\circ} 22^{\prime} / 60^{\circ} 58^{\prime}$,

$16^{\circ} 20^{\prime} / 62^{\circ} 38^{\prime}$

$17^{\circ} 50^{\prime} / 60^{\circ} 48^{\prime}$

$17^{\circ} 58^{\prime} / 64^{\circ} 18^{\prime}$

$18^{\circ} 31^{\prime} / 59^{\circ} 19^{\prime}$

$15^{\circ} 52^{\prime} / 63^{\circ} 22^{\prime}$

$17^{\circ} 50^{\prime} / 60^{\circ} 45^{\prime}$

$17^{\circ} 48^{\prime} / 63^{\circ} 10^{\prime}$

$18^{\circ} 20^{\prime} / 59^{\circ} 50^{\prime}$

$15^{\circ} 55^{\prime} / 61^{\circ} 26^{\prime}$

$18^{\circ} 20^{\prime} / 59^{\circ} 36^{\prime}$

$14^{\circ} 25^{\prime} / 60^{\circ} 50^{\prime}$

$18^{\circ} 10^{\prime} / 64^{\circ} 53^{\prime}$

$19^{\circ} 43^{\prime} / 62^{\circ} 48^{\prime}$,

$18^{\circ} 30^{\prime} / 64^{\circ} 07^{\prime}$

$17^{\circ} 39^{\prime} / 63^{\circ} 08^{\prime}$

$17^{\circ} 24^{\prime} / 63^{\circ} 46^{\prime}$

$19^{\circ} 36^{\prime} / 62^{\circ} 34^{\prime}$

$17^{\circ} 30^{\prime} / 63^{\circ} 10^{\prime}$

COORDinAtes (S/W)

$21^{\circ} 51^{\prime} / 63^{\circ} 40^{\prime}$

$21^{\circ} 56^{\prime} / 64^{\circ} 19^{\prime}$

$21^{\circ} 49^{\prime} / 63^{\circ} 34^{\prime}$,

$21^{\circ} 06^{\prime} / 63^{\circ} 01^{\prime}$,

$20^{\circ} 55^{\prime} / 63^{\circ} 06^{\prime}$

$21^{\circ} 29^{\prime} / 63^{\circ} 13^{\prime}$

$21^{\circ} 41^{\prime} / 65^{\circ} 18^{\prime}$

$21^{\circ} 45^{\prime} / 63^{\circ} 35^{\prime}$

$21^{\circ} 48^{\prime} / 64^{\circ} 14^{\prime}$

$21^{\circ} 15^{\prime} / 63^{\circ} 30^{\prime}$

$19^{\circ} 33^{\prime} / 68^{\circ} 21^{\prime}$

$21^{\circ} 25^{\prime} / 63^{\circ} 09^{\prime}$

$21^{\circ} 05^{\prime} / 63^{\circ} 25^{\prime}$

$21^{\circ} 31^{\prime} / 64^{\circ} 45^{\prime}$

$21^{\circ} 15^{\prime} / 63^{\circ} 30^{\prime}$ 